\title{
Full and partial genome-wide assembly and disassembly of the yeast transcription machinery in response to heat shock
}

\author{
Sara J. Zanton and B. Franklin Pugh ${ }^{1}$ \\ Center for Gene Regulation, Department of Biochemistry and Molecular Biology, The Pennsylvania State University, \\ University Park, Pennsylvania 16802, USA
}

\begin{abstract}
Eukaryotic genes are controlled by sequence-specific DNA-binding proteins, chromatin regulators, general transcription factors, and elongation factors. Here we examine the genome-wide location of representative members of these groups and their redistribution when the Saccharomyces cerevisiae genome is reprogrammed by heat shock. As expected, assembly of active transcription complexes is coupled to eviction of H2A.Z nucleosomes, and disassembly is coupled to the return of nucleosomes. Remarkably, a large number of promoters assemble into partial preinitiation complexes (partial PICs), containing TFIIA, TFIID (and/or SAGA), TFIIB, TFIIE, and TFIIF. However, RNA polymerase II and TFIIH are generally not recruited, and nucleosomes are not displaced. These promoters may be preparing for additional stress that naturally accompany heat stress. For example, we find that oxidative stress, which often occurs with prolonged exposure of cells to high temperature, converts partial PICs into full PICs. Partial PICs therefore represent novel regulated intermediates that assemble at promoters in the midst of chromatin.
\end{abstract}

[Keywords: ChIP-chip; GTF; preinitiation complex; microarrays; Saccharomyces; H2A.Z]

Supplemental material is available at http://www.genesdev.org.

Received April 5, 2006; revised version accepted June 19, 2006.

DNA sequence-specific regulators read the transcriptional regulatory code by binding to promoter elements and orchestrating the assembly and disassembly of the transcription machinery (Levine and Tjian 2003; Harbison et al. 2004). Several key events are connected with transcriptional activation including chromatin remodeling, assembly of the transcription machinery ("PIC" formation), and transcription elongation (Fig. 1A). The core of a PIC, as classically defined in vitro, is an assemblage of general transcription factors (GTFs) and RNA polymerase II (Pol II) on a TATA-box-containing promoter (Reinberg et al. 1998). GTFs include TFIIA, TFIIB, TFIID, TFIIE, TFIIF, and TFIIH. Physiological promoter regions operate in the context of chromatin, which has been suggested to block PIC assembly (Struhl 1999; Narlikar et al. 2002). Gene-specific activators direct chromatin-remodeling/modifying factors to alleviate blocks to PIC assembly and/or transcription (Hassan et al. 2001). They recruit GTFs and enhance Pol II transcription, in some cases through interactions with the mediator complex (Kornberg 2005). The combined interplay of these and

${ }^{1}$ Corresponding author.

E-MAIL bfp2@psu.edu; FAX (814) 863-8595.

Article is online at http://www.genesdev.org/cgi/doi/10.1101/gad.1437506. many other factors contribute to a diverse assemblage of naturally occurring PICs.

PICs assemble via two primary and partially redundant GTF pathways involving either TFIID (TAF-dependent) typically at TATA-less promoters, or SAGA/TBP (TAF-independent) typically at TATA-containing promoters (Kuras et al. 2000; Lee et al. 2000; Li et al. 2000; Cheng et al. 2002; Basehoar et al. 2004; Huisinga and Pugh 2004). The TFIID pathway is central to most genes, whereas the SAGA pathway is particularly tailored for stress-induced gene expression (Basehoar et al. 2004; Huisinga and Pugh 2004).

A clearer picture of the chromatin context in which PICs assemble has recently emerged in Saccharomyces cerevisiae (Ercan et al. 2004; Lieb and Clarke 2005). Many promoters appear to be located within nucleosome-free regions that are flanked by nucleosomes composed of the histone H2A variant H2A.Z (Guillemette et al. 2005; Li et al. 2005; Raisner et al. 2005; Yuan et al. 2005; Zhang et al. 2005). Genes that are highly transcribed appear to have a deficiency of H2A.Z nucleosomes (Guillemette et al. 2005; Zhang et al. 2005). A different study found no correlation between transcription frequency and H2A.Z depletion (Raisner et al. 2005), indicating that the issue may be unresolved. Regardless, 
A

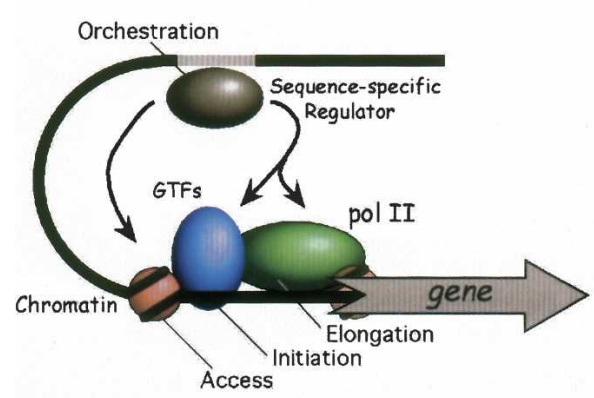

B

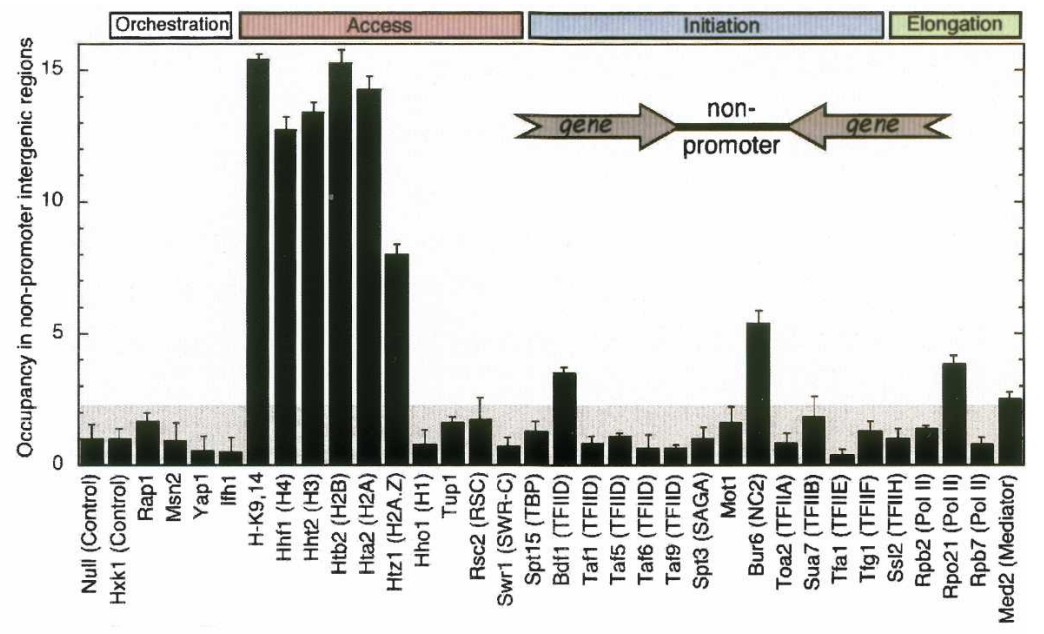

\section{C}

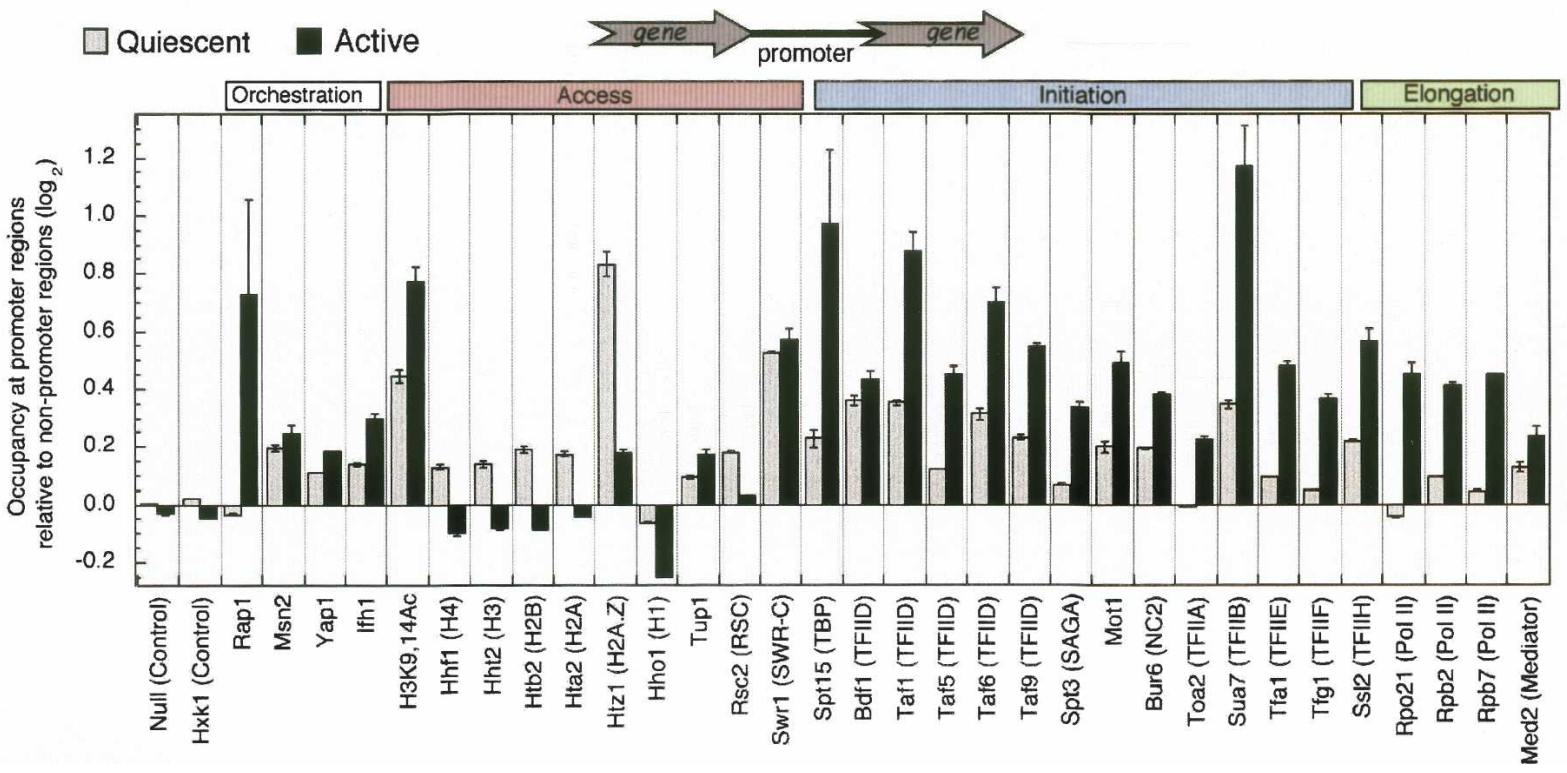

Figure 1. Enrichment of transcription proteins at promoter regions. (A) Schematic of the major stages in eukaryotic transcriptional regulation. Promoter-bound regulators "orchestrate" promoter access via chromatin remodeling, transcription initiation via general transcription factors (GTFs), and transcript elongation via Pol II and its regulators. $(B)$ Genome-wide occupancy levels at nonpromoter regions located between two convergently transcribed genes. Median occupancy levels from combined $25^{\circ} \mathrm{C}$ and $37^{\circ} \mathrm{C}$ data are shown on a linear scale and are relative to null (untagged) or Hxk1 (cytoplasmic protein) control samples. Standard deviations shown here and throughout the paper are drawn from all measured regions in all replicates, and thus reflect a combined region-to-region variance as well as experimental error. The gray background demarcates the null value plus two standard deviations. $(C)$ Occupancy levels at quiescent and constitutively active promoters regions at $25^{\circ} \mathrm{C}$. ChIP data for intergenic regions located upstream of a single gene (i.e., flanking genes are transcribed in the same direction) are shown on a $\log _{2}$ scale relative to nonpromoter regions (i.e., the values generated in $B$ ). Quiescent (gray bars) and active (black bars) promoters are defined as the median occupancy value in the lower 50th percentile (925 genes) and upper 10th percentile (185 genes) of transcription frequencies, respectively, using the data of Holstege et al. (1998).

PIC assembly and/or transcription must contend with chromatin structure at or near promoters.

Many fundamental questions remain regarding the assembly of native PICs within cells. (1) To what extent does each GTF and its regulators populate PICs? (2) Given the observation of nucleosome-free promoter regions, do PICs assemble at promoters unhindered by chromatin? Or are nucleosomes removed either prior to PIC assembly or at some step during assembly?
(3) Does PIC assembly occur in one or multiple regulatable steps? Inasmuch as PIC assembly occurs via multiple pathways involving different components, no single answer may be applicable to all promoters, and thus these questions are best addressed on a genome-wide scale.

The concept of stable PIC intermediates is of particular interest because a variety of such intermediates have been assembled in vitro, yet there is little evidence that 
they exist in vivo as regulated stable intermediates. One widely held view on PIC assembly, based on classical biochemical reconstitution, is that GTFs TFIIA and TBP/TFIID stably assemble onto promoter DNA, which is then followed by stable binding of TFIIB. Next, a Pol II $\bullet$ TFIIF complex is incorporated followed by TFIIE and TFIIH. A few of the many studies that contribute to this view include Buratowski et al. (1989), Conaway et al. (1992), and Flores et al. (1992) and it is reviewed by Orphanides et al. (1996).

Other biochemical experiments suggest alternative assembly steps, including stable incorporation of TFIIE into a promoter complex prior to Pol II entry: (1) TFIIE has been found to cooperatively and stably commit TBP to promoter DNA, which is subsequently bound by Pol II (Conaway et al. 1990). (2) Tjian and colleagues report that TFIIE binds to and stabilizes TBP • TATA and TFIIA • TBP • TATA complexes in the absence of Pol II, leading them to propose an alternative PIC assembly intermediate (Yokomori et al. 1998). (3) Archaeal TFIIE also stabilizes TBP/TATA interactions in the absence of Pol II (Bell et al. 2001). (4) Isolated TFIIA, TFIIB, and TFIID subunits directly contact either TFIIE or TFIIF in the absence of Pol II (Ha et al. 1993; Ruppert and Tjian 1995; Dubrovskaya et al. 1996; Fang and Burton 1996; Langelier et al. 2001). (5) Numerous gene-specific transcriptional activators including Fos/Jun, Antp, p100, Kruppel, androgen receptor, and SRF directly contact either TFIIE or TFIIF in the absence of Pol II (Joliot et al. 1995; Sauer et al. 1995; Tong et al. 1995; Martin et al. 1996; Zhu and Kuziora 1996; McEwan and Gustafsson 1997). Taken together, in vitro biochemical evidence supports the idea that TFIIE and TFIIF may be recruited to promoters independently of Pol II or in conjunction with Pol II. Here we present in vivo evidence for the genome-wide assembly of TFIIE and TFIIF into a PIC with and without Pol II. The idea that GTFs can be recruited to promoters in the absence of Pol II in vivo has precedent in that several studies find that TBP or the Pol II-associated Mediator can be recruited to certain promoters without Pol II (Bhaumik and Green 2001; Cosma et al. 2001; Bryant and Ptashne 2003; Fan et al. 2006).

To investigate the extent to which a wide variety of transcription proteins come and go at promoters, we have measured the genome-wide redistribution of gene regulatory proteins when the yeast genome is reprogrammed by heat stress. Thirty-two proteins involved in regulating various stages of gene regulation were examined. These include sequence-specific DNA-binding proteins, chromatin proteins and their regulators, and the GTFs. Comparisons of these data reveal a genome in which full transcriptionally active PIC assembly is highly coordinated and directly linked to nucleosome disassembly. Strikingly, many promoters undergo partial PIC assembly in which Pol II is not recruited, nucleosomes are not lost, and the gene is not activated. Many partial PICs can be driven into full PICs when exposed to an additional signaling event. Together these findings present the first evidence for the widespread assembly of regulated PIC intermediates that assemble at promoters in the midst of chromatin.

\section{Results}

Genome-wide occupancy of TAP-tagged transcription proteins were measured by chromatin immunoprecipitation assays coupled to microarray detection (ChIP-chip). Occupancy levels were measured in rich media at $25^{\circ} \mathrm{C}$, then changes in occupancy were measured after a $15-\mathrm{min}$ heat shock at $37^{\circ} \mathrm{C}$. Heat shock results in the transcriptional reprogramming of at least $10 \%$ of the yeast genome (Gasch et al. 2000; Causton et al. 2001). Heat shock provides an experimentally ideal stimulus for genomic reprogramming because it mobilizes the two major PIC assembly pathways, involving TFIID and SAGA. Data quality was assessed and validated as described in the Supplemental Material. Processed ChIP-chip data are reported in Supplementary Table S1.

\section{Enrichment of transcription proteins at promoter regions}

In this study, we expected to observe promoter-selective binding of sequence-specific regulators as well as nonselective binding of broadly acting factors such as histones. Nonselective binding should be manifested by occupancy in all regions including nonpromoter regions, such as the intergenic regions between two convergently transcribed genes. As a first step toward assessing selectivity, we first measured factor occupancy in nonpromoter regions. Figure 1B displays a bar graph representation of the median transcription factor occupancy at $>2000$ intergenic nonpromoter regions. As expected, all four core histones (H2A, H2B, H3, H4) were present, including a moderate amount of H2A.Z. Most other transcription proteins appeared to be generally absent from nonpromoter regions.

We then measured bulk occupancy at promoter regions relative to nonpromoter regions (Fig. 1C), distinguishing between transcriptionally quiescent $(<1$ mRNA/ h) and active (>14 mRNA/h) promoters (Holstege et al. 1998). Most proteins involved in transcription initiation or elongation such as TFIIB, TFIID, and Pol II displayed greater enrichment at active promoters, consistent with the general view that PICs assemble at active genes. The four core histones were relatively depleted at active promoters compared with quiescent promoters, consistent with recent reports (Bernstein et al. 2004; Boeger et al. 2004; Lee et al. 2004). Relative to nonpromoter regions, H2A.Z was highly enriched at most promoter regions that were transcriptionally quiescent, and comparatively depleted at highly transcribed genes. This is in agreement with recent reports suggesting that H2A.Z is an epigenetic chromatin mark of promoter regions (Guillemette et al. 2005; Li et al. 2005; Raisner et al. 2005; Zhang et al. 2005). The significance of low amounts of H2A.Z in nonpromoter regions remains to be explained. The general adherence of the data to the prevailing view on occupancy levels expected at active versus quiescent promoters provides further validation that 
these ChIP-chip assays measure true genome-wide occupancy levels.

\section{PIC assembly and disassembly is highly coordinated throughout the genome}

In the next phase of the analysis, we examined the redistribution of the transcription machinery when the yeast genome is reprogrammed by heat shock (sudden shift from $25^{\circ} \mathrm{C}$ to $37^{\circ} \mathrm{C}$ ). Figure 2 (left panel) is a cluster plot representation of changes in occupancy on a geneby-gene basis for a variety of gene regulatory proteins, and reveals several general relationships. First, changes in occupancy of H2A.Z and all four core histones, except an acetylated form of $\mathrm{H} 3$, were tightly linked to each other as reflected in the shallow branching pattern (shown in red) of the dendrogram relating occupancy changes of the various proteins. Second, a deep division in the dendrogram is evident between the histones and PIC components (red vs. blue and green branches), reflecting a highly coordinated change in occupancy in opposing directions. Third, the GTFs (except TFIIH) and their regulators behaved similarly (blue branches), reflecting their combined presence in a PIC. Fourth, the behaviors of TFIIH and Pol II (green branches) were, in general, more similar to each other and less similar to other GTFs, which is consistent with TFIIH being a modulator of Pol II activity. Fifth, changes in Pol II occupancy largely mirrored changes in mRNA levels (Fig. 2 , right panel), indicating that Pol II recruitment is generally associated with changes in gene expression. This "bird's eye" view reveals a highly coordinated pattern of assembly and disassembly of a wide range of PIC components and its regulators (Fig. 2, schematic).
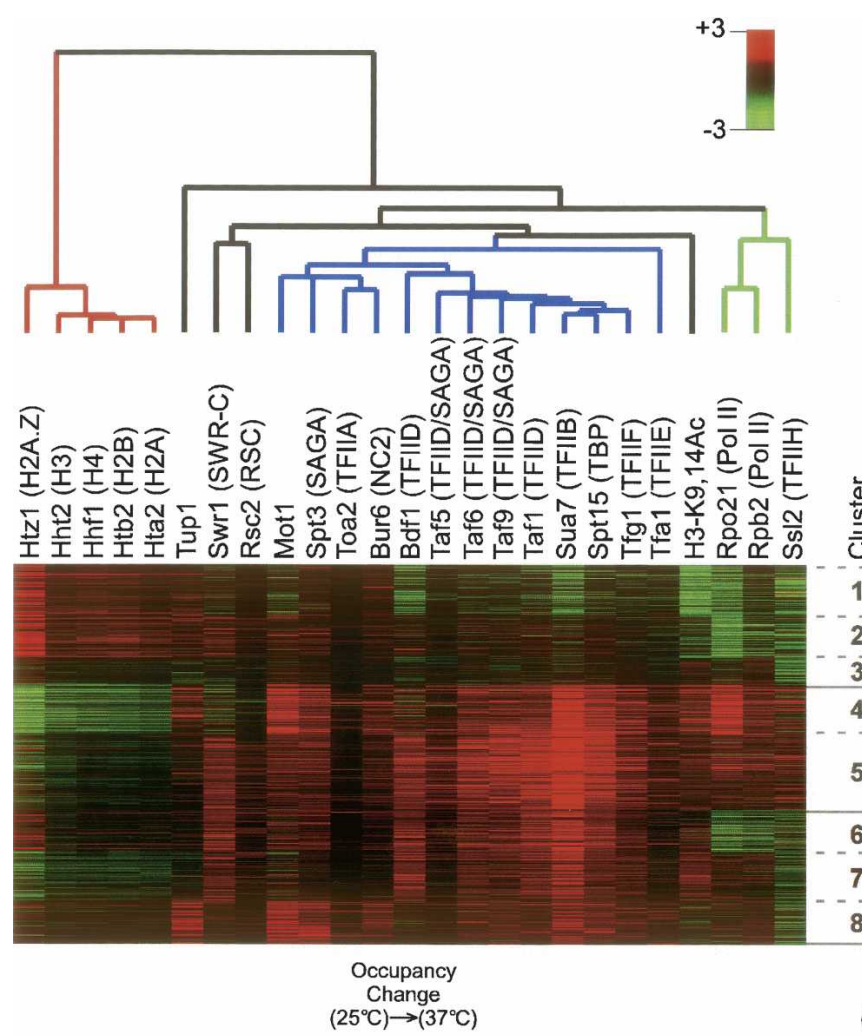

3

5

5

$\frac{6}{7}$

7

Change
$\left.5^{\circ} \mathrm{C}\right) \rightarrow\left(37^{\circ} \mathrm{C}\right)$

Expressio

Change
$\left.5^{\circ} \mathrm{C}\right) \rightarrow\left(37^{\circ} \mathrm{C}\right)$

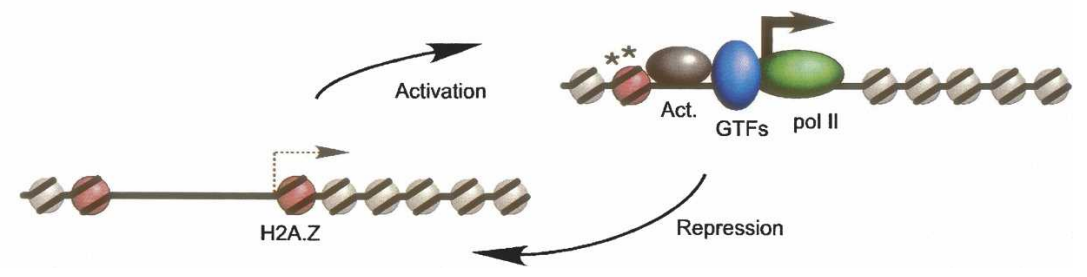

Figure 2. PIC assembly and disassembly are highly coordinated throughout the genome. In the left panel, $\log _{2}$ ratios of $37^{\circ} \mathrm{C} / 25^{\circ} \mathrm{C}$ occupancy changes for intergenic regions that are immediately upstream of a single gene were filtered to obtain values that increased (red) or decreased (green) the most (defined as being in the upper or lower 10th percentile in any one data set, and thus do not represent absolute cutoffs). Black denotes no change. A total of 1952 intergenic regions ( $72 \%$ of the analyzed genome) met these criteria. Since the variance in each column was not normalized, the intensity of the color changes across different columns have not accounted for differences in cross-linking efficiency. Rows representing intergenic regions were clustered by $K$-means $(K=9)$ (Eisen et al. 1998). Only eight clusters are shown since the ninth cluster corresponded to changes in Pol II and TFIIH occupancy occurring predominantly at the $3^{\prime}$ end of the adjacent upstream gene rather than at the $5^{\prime}$ end of the downstream gene. Columns representing individual ChIP-chip experiments were clustered hierarchically. The dendrogram relating them is color-coded according to the broad classifications illustrated in Figure 1A. In the right panel, corresponding changes in gene expression (mRNA levels) are shown, using the same color code as in the left panel. Median transcription frequencies in mRNA per hour (Holstege et al. 1998), and median fold changes in mRNA levels (non-log scale) for each cluster are indicated. The expression data were not used in filtering or clustering. "Mock" denotes a mock temperature shift. The schematic illustrates a simplified model depicting the cycle of PIC assembly and disassembly during heat-shock activation and repression. Asterisks reflect histone acetylation. 
Several factors contributed to the median fold change in gene expression for each cluster being below the maximal expected heat-shock response. First, our ChIP-chip filtering criteria are less stringent than imposed previously on expression data (Gasch et al. 2000; Causton et al. 2001), resulting in a greater number of modest fold changes being included. Second, we report median fold changes in expression for clusters whose membership is derived from a diverse set of ChIP-chip data rather than from expression data. Cluster membership required that only a single Chip-chip value from 25 data sets be in the upper or lower 10 percentile of its data set. Thus, there was no direct selection for genes whose expression responded maximally to heat shock. Third, many changes in occupancy, which drive the clustering patterns, are not coupled to changes in gene expression. These lack of changes diminished the median expression values for each cluster. Nevertheless, as shown in Supplementary Figure S2, the magnitude of change in gene expression that we observed in response to heat shock and the identity of responding genes were essentially identical to that observed previously (Gasch et al. 2000; Causton et al. 2001).

\section{PIC assembly leads to nucleosome depletion upon transcription}

The inverse relationship between nucleosome and PIC formation was very strong as evident by quantitation of H2A.Z (Htz1) and Pol II (Rpo21/Rpb1) occupancy changes for $>5300$ loci $(R=0.74)$ (Fig. 3A, left panel). $R$ increased to 0.9 when loci that were not undergoing significant changes in H2A.Z or Pol II occupancy were excluded (data not shown). Similar results were obtained when other histones and other subunits of Pol II (Rpb2 and Rpb7) were compared (data not shown), but not when an untagged "null" control was plotted $(R=0.1)$ (Fig. 3A, right panel). These findings represent the first quantitative demonstration that, almost without exception, whenever transcriptionally active Pol II is recruited to promoter regions, H2A.Z nucleosomes are displaced. When Pol II departs, H2A.Z nucleosomes return. These findings support related studies showing a trend between nucleosome depletion and transcription (Bernstein et al. 2004; Lee et al. 2004; Schwabish and Struhl 2004; Guillemette et al. 2005; Zhang et al. 2005). However, a direct comparison between changes in occupancy of H2A.Z and Pol II, as shown here, reveals a much more robust relationship than was previously evident.

Since Pol II binding to promoter regions is linked to transcription, neither the current study nor any previous study could determine whether nucleosome disruption in promoter regions was necessary for PIC assembly or whether the act of transcription displaced nucleosomes. To address whether H2A.Z is removed from promoter regions by Pol II recruitment versus Pol II transcription, we shut down transcription using a temperature-sensitive $r p b 1-1$ (rpo21) strain that rapidly ceases mRNA synthesis upon heat shock (Nonet et al. 1987). Mutant Pol II was not noticeably degraded under such conditions (data not shown). Due to the loss of Pol II activity, it is unlikely that these cells are mounting any sort of normal heat-shock response, and thus caution should be applied if comparisons are made to wild-type strains.

We needed to first establish whether the mutant Pol II was still capable of binding promoter DNA when inactivated by heat shock. Therefore, ChIP-chip was performed with the Rpb2 subunit of Pol II under standard rpb1-1 permissive $\left(25^{\circ} \mathrm{C}\right)$ and nonpermissive (45-min shift to $37^{\circ} \mathrm{C}$ ) (Holstege et al. 1998) conditions. Changes in occupancy were determined. To verify that the changes reflected changes in occupancy, as opposed to changes in nonspecific background, we also measured occupancy changes for TFIIE and TFIIF, which should mirror Pol II, and for an untagged "null" control, which should provide a measure of nonspecific background. Correlation plots of these factors with $\mathrm{Rpb} 2$ are shown in Figure 3B. Changes in mutant Pol II occupancy correlated with changes in TFIIE and TFIIF occupancy $(R>0.7)$ and less so with the untagged control $(R=0.4$, with substantially fewer data points having a signal above the local background). This suggests that in the absence of Pol II transcription, these GTFs and the mutant Pol II could still coordinately bind to promoter regions. We next examined the correlation between Rpb2 and the two histones H2A.Z and H2A (Fig. 3B). Little or no correlation was observed relative to the untagged control, suggesting that transcription rather than Pol II recruitment or PIC assembly is a primary contributor to nucleosome eviction in promoter regions. Similar conclusions have been made at the human $\alpha 1$ antitrypsin promoter (Soutoglou and Talianidis 2002). Our result does not rule out a minority of cases in which nucleosome disruption might precede PIC assembly.

Sequence-specific regulators relocate upon heat shock without necessarily altering nucleosome occupancy

Several clusters generated in Figure 2 (e.g., clusters 3, 5-8) showed novel patterns of assembly and disassembly of PIC components that did not lead to significant changes in gene expression. To better understand these phenomena including how functionally related transcription proteins coordinate their movement throughout the genome during heat shock, we focused on selected proteins. Reclustering of ChIP profiles on selected proteins allowed patterns that were dispersed over multiple clusters in Figure 2, and thus overlooked, to become visually more apparent. We first examined sequence-specific regulators, then chromatin factors, and finally the GTFs.

The genome-wide distribution and relocation upon heat shock of five sequence-specific transcriptional regulators of stress-response genes are presented in Figure 4A. At $25^{\circ} \mathrm{C}$, Rapl occupied the promoters of genes in cluster 11 (Fig. 4A, column 5). These genes were highly expressed (Fig. 4A, column 8), and had a large representation of ribosomal protein genes, as expected (Lieb et al. 2001). Surprisingly, upon heat shock, additional Rap1 accumulated (Fig. 4A, column 13), despite gaining nucleo- 
A

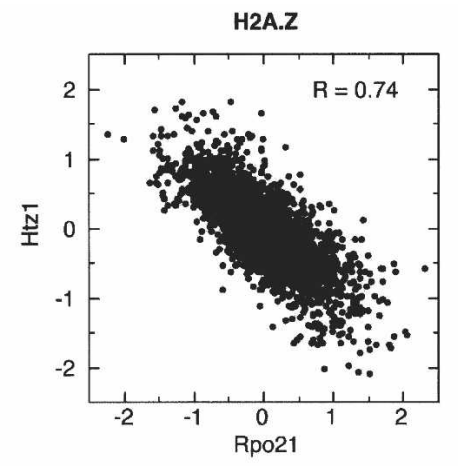

B

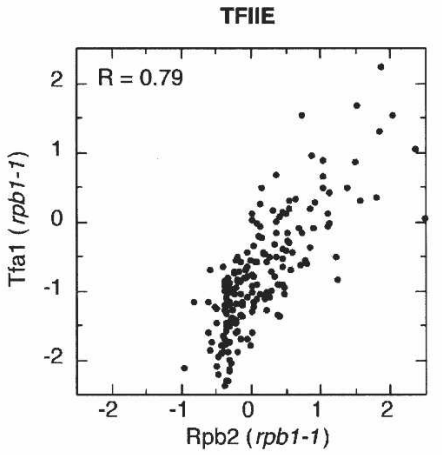

H2A.Z

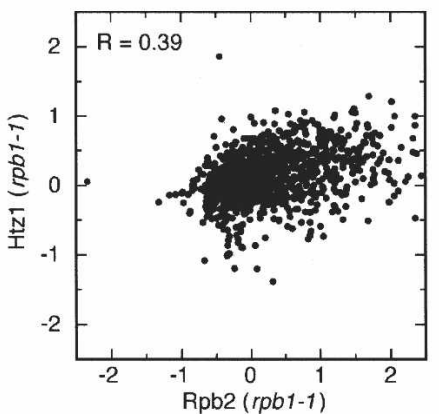

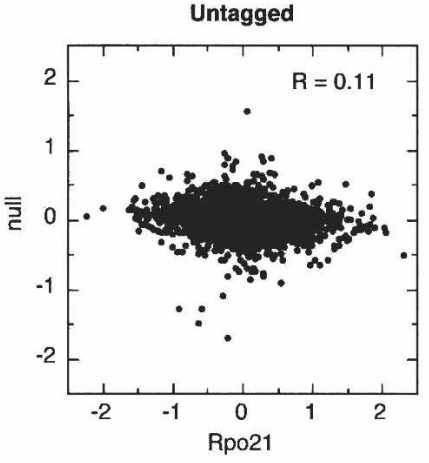

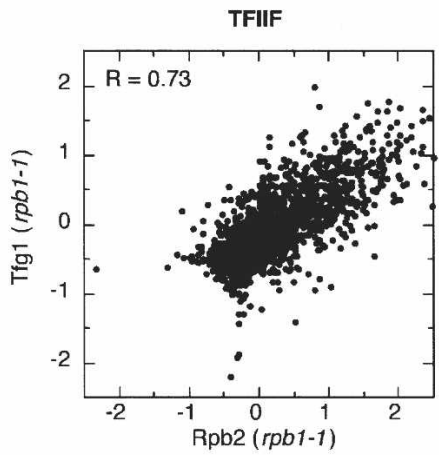

H2A

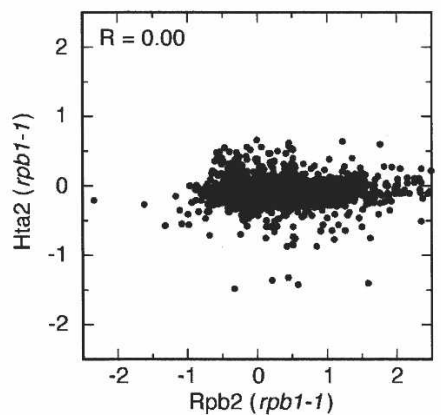

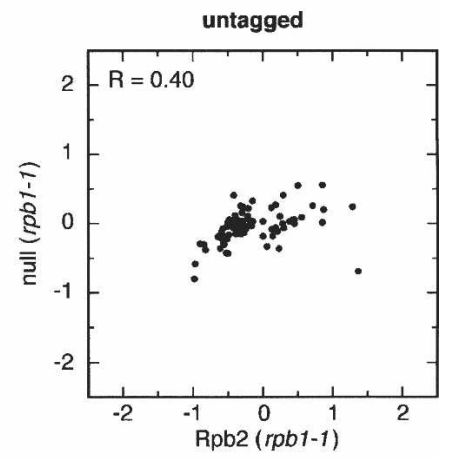

Figure 3. Changes in histone occupancy as a function of changes in Pol II occupancy. (A) In the left panel, $\log _{2}$ fold changes in Htzl (H2A.Z) occupancy at 5379 intergenic regions are plotted as a function of Rpo21 (Pol II) occupancy changes upon heat shock $\left(25^{\circ} \mathrm{C} \rightarrow 37^{\circ} \mathrm{C}\right.$ for $\left.15 \mathrm{~min}\right)$. In the right panel, equivalent data from an untagged (null) control strain are plotted. $(B) \log _{2}$ fold changes in occupancy of TFIIE, TFIIF, H2A.Z, H2A, and an untagged control in a temperature-sensitive rpb1-1 strain are plotted as a function of Pol II (Rpb2) occupancy changes upon heat inactivation of Pol II $\left(25^{\circ} \mathrm{C} \rightarrow 37^{\circ} \mathrm{C}\right.$ for $\left.45 \mathrm{~min}\right)$. The variable number of data points in each graph reflects the number of measurements that were above the local microarray background in both replicates (see Materials and Methods).

somes (Htz1, Fig. 4A, column 9), losing Pol II (Rpo21) Rpb1, Fig. 4A, column 15), and decreasing in expression (Fig. 4A, column 16). Thus, while Rap1 normally activates ribosomal protein genes, higher levels are linked to inhibition, which is consistent with the finding that multiple Rap1-binding sites confer transcriptional inhibition in response to heat shock (Li et al. 1999). This represents one example where additional binding of a sequence-specific transcriptional activator is associated with increased nucleosome occupancy and transcriptional repression.

Another redistribution pattern of stress-response activators, represented by cluster 12 (Fig. 4A), reveals a loss of Msn2 (Fig. 4A, column 10) and an acquisition of Yap1
(Fig. 4A, column 11) and Ifh1 (Fig. 4A, column 12) upon heat shock. However, genes in this cluster displayed little or no change in expression (Fig. 4A, column 16), and only minor alterations in H2A.Z, Pol II, or core histone occupancy (Fig. 4A, columns 9,15; data not shown). The promoters in cluster 12 also had the atypical property of having high occupancy of a variety of other sequence-specific DNA-binding regulators that have been implicated in cell cycle control, sporulation, and oxidative stress. These include Yap5, Ace2, Aft2, Rcs1, Ime4, Mbp1, Ndd1, and Swi5 ( $p<10^{-5}$, which is based on data from Lee et al. [2002] and Harbison et al. [2004], presented in Supplementary Table S2 as entries 1231, 1334, $1519,1541,1601,1637,1649$, and 1687). It is an intrigu- 
A

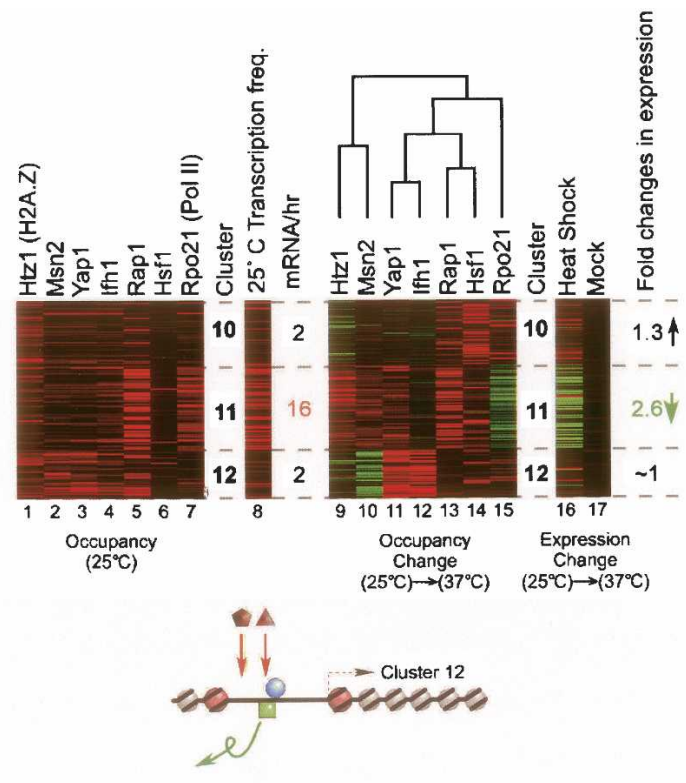

B

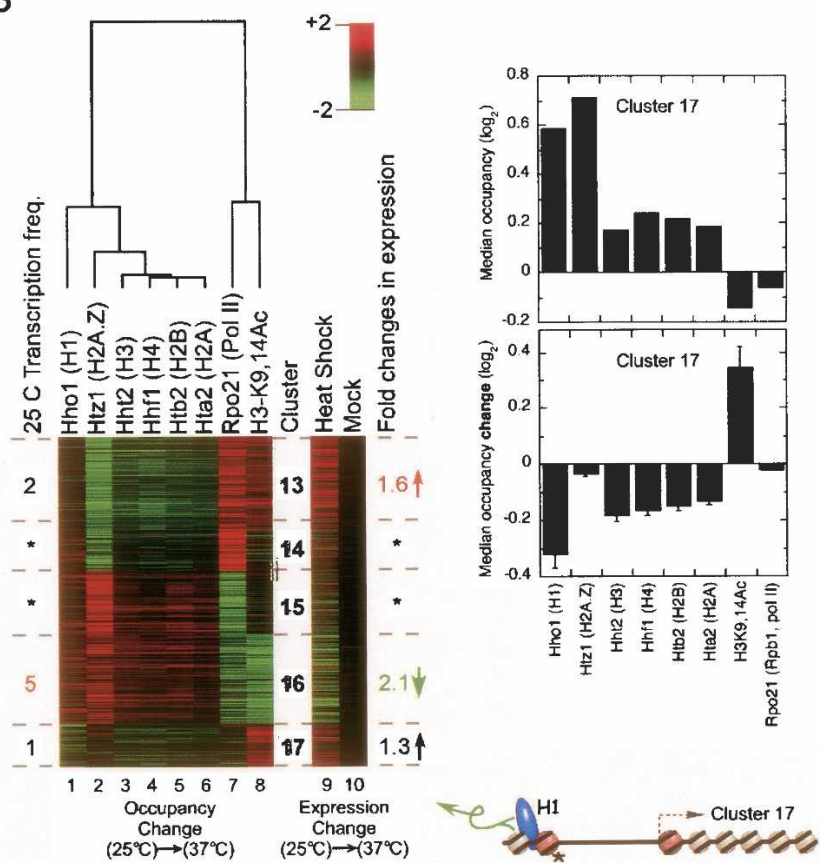

Figure 4. Redistribution of selected transcriptional regulators upon heat shock. $(A)$ Redistribution of sequence-specific regulators. The left cluster plot (columns 1-7) shows occupancy levels at $25^{\circ} \mathrm{C}$, where brighter red reflects higher occupancy and black is relatively low occupancy. Column 8 displays the corresponding transcription frequency ( $\log _{10}$ scale in mRNA per hour) at $25^{\circ} \mathrm{C}$. Median non-log transcription frequency values for each cluster are indicated. Columns 9-15 are corresponding changes in occupancy after a 15-min temperature shift from $25^{\circ} \mathrm{C}$ to $37^{\circ} \mathrm{C}$, as described in Figure 2. Intergenic data for the indicated proteins were filtered as described in Figure 2, using a 2 percentile cutoff for increased occupancy for Yap1, Msn2, and Ifh1; a 2 percentile cutoff for decreased occupancy for Msn2 and Ifh1; a 3 percentile cutoff for increased occupancy of Hsf1 (Hahn et al. 2004); and a 10 percentile cutoff for increased occupancy of Rap1. A total of 213 intergenic regions ( $8 \%$ of the analyzed genome) met these criteria. We found no evidence for any significant decrease in occupancy for Rap1 and Hsf1 (Hahn et al. 2004). These filtering criteria were applied only to occupancy changes for Msn2, Hsf1, Rap1, Ifh1, and Yap1 in columns 10-14. The corresponding data in columns 1-9 and 15-17 were subsequently added. Thus, all row data are linked across columns $1-17$. All data in columns 9-15 were used in $K$-means $(K=3)$ clustering of rows, and were variance normalized (Eisen et al. 1998). The magnitude of the color scale is therefore arbitrary, although $\log _{2} \operatorname{linear}_{\text {. Columns } 16 \text { and }}$ 17 display $\log _{2}$ changes in gene expression. Median non-log values for each cluster are also reported. Below the cluster plots is a model conceptualizing the data from cluster 12, where sequence-specific regulators (shown in color) bind to (red arrows) or dissociate from (green arrow) promoters without concomitant changes in nucleosome occupancy or PIC assembly. (B) Redistribution of histones upon heat shock. Data in columns 1-8 were filtered at the 10 percentile level as described in Figure 2, and clustered into five groups (13-17). A total of 1095 intergenic regions $(40 \%)$ passed this filtering criteria. Data were not variance normalized. Clusters 14 and 15 are influenced by an overabundance of structural RNA genes (tRNA, snRNA, and snoRNA; $p \sim 10^{-7}-10^{-10}$ ) present in these intergenic regions, and thus were not further analyzed here. Columns 9 and 10 display changes in mRNA levels. The upper and lower bar graphs, respectively, display median occupancy at $25^{\circ} \mathrm{C}$ and median fold changes in occupancy $\left(25^{\circ} \mathrm{C} \rightarrow 37^{\circ} \mathrm{C}, 15 \mathrm{~min}\right)$ in cluster 17 for the indicated proteins. The lower apparent occupancy level of the core histones relative to H2A.Z (upper bar graph) is likely due to the fact that $S$. cerevisiae possesses two genes for each core histone, of which only one is TAP-tagged. Below the graphs is a model illustrating the heat-shock mobilization of Hhol (H1), shown in blue, at cluster 17 genes. Its precise location is uncertain. As shown, dissociation of $\mathrm{H} 1$ and increased $\mathrm{H} 3$ acetylation $\left({ }^{\star}\right)$ are not concomitant with Pol II recruitment, and thus are likely to occur prior to full PIC assembly at these genes.

ing possibility that the genes in cluster 12 might be poised to alter the cell cycle and thus cell growth rate if exposed to additional stress. The pattern of occupancy change reflected by cluster 12 suggests that net association or dissociation of sequence-specific regulators at certain promoters occurs in a manner that is highly coordinated but largely uncoupled from changes in Pol II or nucleosome occupancy or changes in gene expression (Fig. 4A, diagram). This is the first piece of evidence that we present suggesting an assemblage of transcription components at some promoters without a commitment to full PIC assembly (as measured by Rpo21/Rpb1 incorporation) or changes in transcription.

\section{The histone H1 homolog Hho1 dissociates from repressed promoter regions upon heat shock without concomitant gene activation}

Hhol is the putative ortholog of linker histone H1 that establishes higher-order chromatin structure in multicellular eukaryotes. Biochemically, Hhol binds to nucleosomes (Patterton et al. 1998) and is expressed dur- 
ing S phase coordinately with the core histones (Spellman et al. 1998). Yet its functional association with histones in vivo has been elusive. Of all the proteins tested, none generated a ChIP pattern that was more similar to the four core histones and H2A.Z than Hhol (Fig. 4B, cluster plot). Hhol generally came and went in concert with the core histones, although its changes in occupancy were typically less robust, as might be expected of its relatively low genome-wide abundance (Freidkin and Katcoff 2001). This linkage to the core histones provides in vivo support to the notion that Hhol is histone $\mathrm{H} 1$ that binds to yeast nucleosomes.

Cluster 17 showed an intriguing relationship between histone $\mathrm{H} 1$ and the core histones. Compared with other promoters at $25^{\circ} \mathrm{C}$, the promoter regions in cluster 17 had high $\mathrm{H} 1$ levels, normal H2A.Z levels, and were underacetylated at H3K9,14 (Fig. 4B, upper bar graph). They were also among the most quiescent genes throughout the genome, being inhibited by a variety of chromatin regulators including Set1, Tup1, Rpd3, and histone $\mathrm{H} 3$ / $\mathrm{H} 4$ tails $\left(p<10^{-10}\right.$, which is based on data derived from references listed in Supplementary Table S2, entries 252, $990,242,456$, and 458). As quantified in the lower bar graph in Figure $4 \mathrm{~B}, \mathrm{H1}$ and a moderate amount of the core histones dissociated from cluster 17 intergenic regions upon heat shock, while at least some remaining $\mathrm{H} 3$ increased in acetylation. However, H2A.Z did not depart, Pol II was not recruited, and these genes were not substantially turned on. Thus, the chromatin at the promoter regions in cluster 17 appears to be undergoing some transformation that is linked to the loss of $\mathrm{H} 1$ and perhaps some limited nucleosome disassembly, but not linked to the assembly of a Pol II-containing PIC or loss of H2A.Z nucleosomes (Fig. 4B, schematic). These results show that $\mathrm{H} 1$ dissociates from some genes during heat shock, but these genes are not activated during the time frame of a typical heat-shock response, thereby providing one example of a specific type of chromatin remodeling without concomitant PIC assembly or transcription. This remodeling does not appear to involve H2A.Z nucleosomes.

\section{TFIIA, TFIID/SAGA, TFIIB, TFIIE, and TFIIF assemble into partial PICs in vivo without TFIIH and Pol II}

The composition of native cellular PICs is largely unknown. In fact, it is not known whether all PICs that assemble in vivo contain every GTF. Genome-wide expression profiling of GTF mutants suggests that at least some genes do not depend on certain GTFs (Holstege et al. 1998; Lee et al. 2000).

To examine PIC assembly and disassembly in more detail, we focused on the redistribution of GTFs in response to heat shock (Fig. 5A). Promoters in cluster 21, representing nearly $8 \%$ of the measured genome, assembled Spt15 (TBP), Sua7 (TFIIB), Tfa1 (TFIIE), and Tfg1 (TFIIF) in response to heat shock (Fig. 5A, columns 2-5). Strikingly, Ss12 (TFIIH) and Rpo21 (Pol II) did not appreciably assemble (Fig. 5A, columns 6,7), H2A.Z did not dissociate (Fig. 5A, column 1), and the corresponding genes were not induced (Fig. 5A, column 8). The same trend was observed when individual genes were examined by standard ChIP assays (Fig. 5B), thereby reconfirming these findings with an assay that does not involve microarrays. To further substantiate GTF assembly in the absence of Pol II recruitment, we produced ChIP-chip data for additional PIC components. In Figure 5C, the median change in GTF and Pol II occupancy for the 205 genes that comprise cluster 21 is plotted. In addition to these GTFs, we report measurements for four TAFs (Tafs 1, 5, 6, and 9), one additional GTF (TFIIA/ Toa1), two SAGA-related components (Spt3 and Mot1), two additional Pol II subunits (Rpb2 and $\mathrm{Rpb} 7)$, and three histones (H2A.Z/Htz1, H4, and H3K9,14,Ac), none of which were used in defining cluster 21 . The bar graph reveals a statistically significant enrichment of the GTFs TFIIA, TFIIB, TFIID, TFIIE, TFIIF, and SAGA, but no enrichment of any of the three Pol II subunits or TFIIH (Ssl2). There was no change in nucleosome occupancy, and no change in transcription. Many of the same trends were evident in clusters 8,12 , and 17 , which also underwent remodeling in the absence of transcription (data not shown). The broadness of our coverage of GTFs, Pol II, and histones make it statistically improbable that these effects could have arisen from anomalous differential cross-linking of proteins to promoters. The clear break between GTF recruitment and Pol II recruitment reveals that in response to heat shock, many promoters undergo partial PIC assembly in the midst of chromatin. Most GTFs are recruited, but TFIIH and Pol II are excluded. These GTFs are unlikely to be the sole components of partial PICs. They are likely to contain DNA sequence-specific regulatory proteins and other components that have yet to be defined.

As introduced earlier, there is ample biochemical evidence for partial PIC assembly. TFIIA, TFIIB, TFIID, TFIIE form stable complexes with promoter DNA in vitro in the absence of Pol II and TFIIH (Conaway et al. 1990; Yokomori et al. 1998; Bell et al. 2001). These GTFs also make direct contact with each other and with sequence-specific activators in the absence of Pol II and TFIIH. However, TFIIF has been firmly established as being Pol II associated, and responsible for escorting Pol II to promoters (Flores et al. 1991; Killeen et al. 1992). This is in apparent contrast to our findings that TFIIF resides in a partial PIC in the absence of Pol II. Nonetheless, our findings do not exclude the possibility that TFIIF (or any other factor in the partial PIC) must dissociate prior to full PIC assembly, then reassociate in a different form such as a Pol II • F complex.

Taken together, these results suggest that in addition to full PIC assembly as seen in cluster 20 (Fig. 5A), many other promoter regions undergo partial PIC assembly as evident in cluster 21. Other promoter regions /cluster 18), which include many of the ribosomal protein promoters, underwent full PIC disassembly in response to heat shock. Astonishingly, cluster 19 underwent partial disassembly, proceeding about as far as the partial assembly in cluster 21. Cluster 19 lost primarily Ss12 (TFIIH) and Rpo21 (Pol II) and gained H2A.Z. Genes in 
A

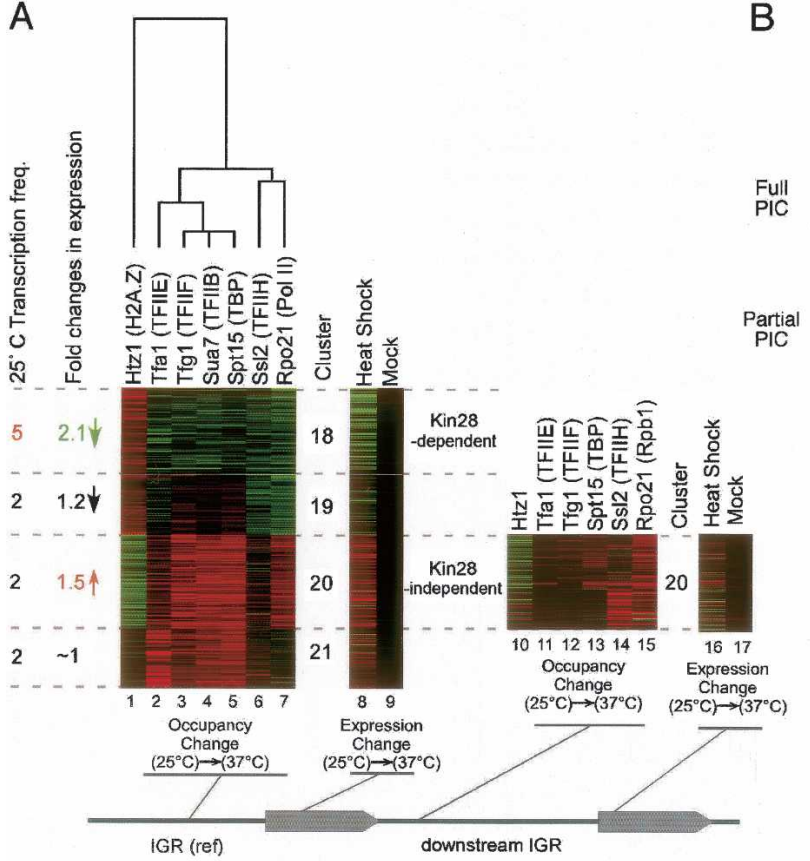

B
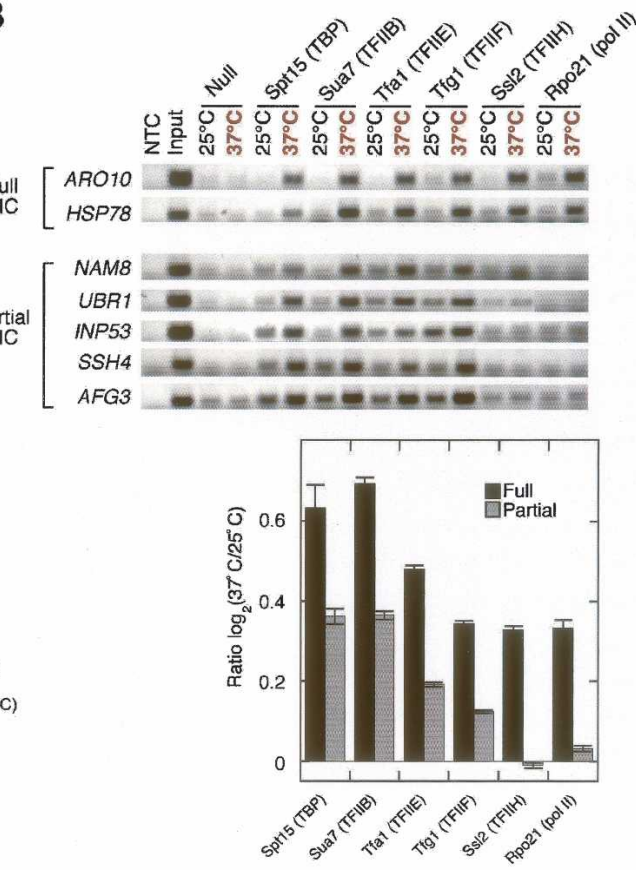

C

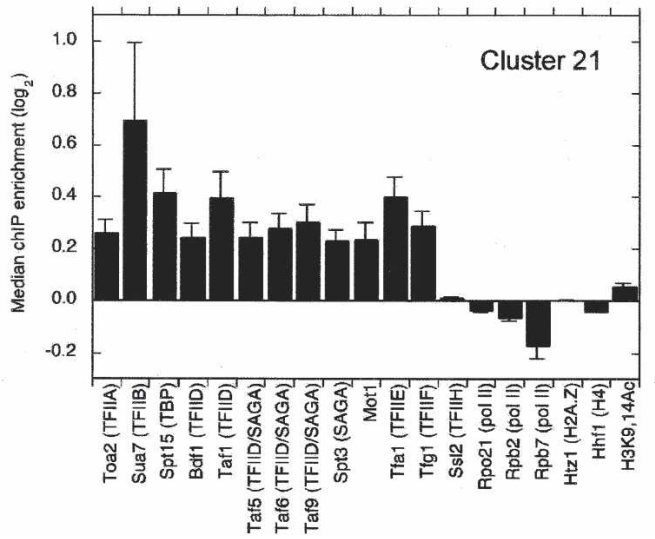

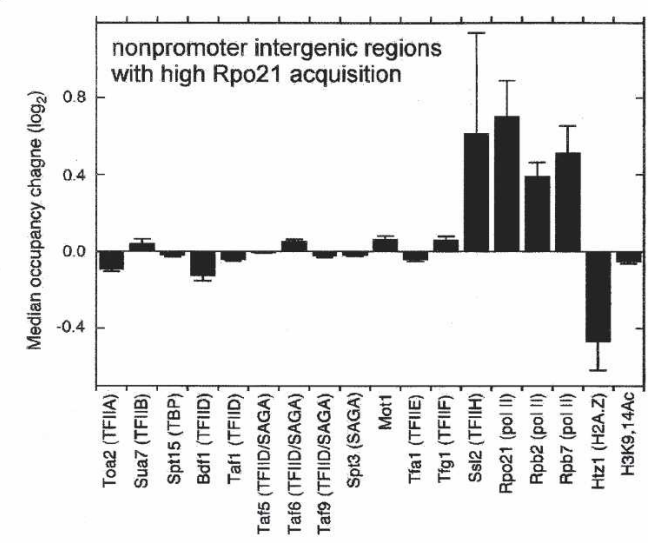

Figure 5. Partial PIC assembly. (A) Redistribution of GTFs in response to heat shock. Occupancy changes were filtered at the 10 percentile level, clustered by $K$-means into five groups (18-22), and variance normalized, resulting in an arbitrary $\log _{2}$ color scale. A total of 1197 intergenic regions (44\%) passed this filtering criteria. The Htz1 (H2A.Z) data (column 1) were not used for filtering, but were subsequently reattached. Cluster 22 is not displayed since it corresponded to Rpo21 and Ssl2 binding at the 3' end of the adjacent upstream gene rather than in promoter regions. Cluster 20 was further subclustered $(K=2)$ using only the ChIP-chip data in columns 10-15, which represent occupancy changes occurring in the intergenic region (IGR; see diagram) immediately downstream from the corresponding cluster 20 gene. Changes in gene expression are displayed in columns 8-9 and 16-17. (B) Standard ChIP analysis demonstrating partial PIC assembly. Samples were prepared as for ChIP-chip as in A except that gene-specific primers were used to PCR-amplify the promoter regions of the indicated genes (see Supplemental Material). Full PIC assembly genes were chosen from cluster 4 (Fig. 2). Partial PIC assembly genes were chosen from clusters 6, 7, and 21. Shown are representative ethidium-bromidestained gels in which the PCR products are in the linear response range. At least five independent replicates were performed for each experiment, and representative data are shown. Template DNA was omitted in the lane marked "NTC." Due to differences in protein-specific and gene-specific cross-linking efficiencies, only matched pairs of $37^{\circ} \mathrm{C}$ and $25^{\circ} \mathrm{C}$ data can be compared. To provide a summarized measure of the fold changes in occupancy upon heat shock, $\log _{2}$ ratios for all predicted partial PIC complexes were averaged and plotted as a bar graph (gray bars). The same was done for those predicted to undergo full PIC assembly (black bars). Quantitation and error values for individual genes are presented in Supplementary Figure S3. (C) Bar graph quantitation of the median $\log _{2}$ occupancy changes for the indicated factors at the promoter region in cluster 21 (shown in $A$ ). Other core histones behaved equivalently to $\mathrm{H} 4$ (data not shown). (D) Bar graph quantitation of the median $\log _{2}$ occupancy changes for all nonpromoter intergenic regions (i.e., downstream from two convergently transcribed genes) that have a high-level Rpo21 acquisition (defined as being in upper 10 percentile). The large standard deviation for TFIIH is consistent with the observation in $A$ that TFIIH does not associate with some Pol II complexes. 
clusters 19 and 21 were not biased toward either the TFIID or SAGA assembly pathway (entry 562 in Supplementary Table S2), indicating that this phenomenon is applicable to both pathways. Taken together, these findings reveal that the same basic partial PIC can be achieved by either partial assembly or partial disassembly.

Partial PICs appear to be widespread, and might represent novel and potentially regulated PIC intermediates in a stepwise assembly of the transcription machinery. Moreover, when these partial PICs assemble they generally do not displace $\mathrm{H} 2 \mathrm{~A}$.Z nucleosomes or alter their $\mathrm{H} 3$ K9,14 acetylation status (Fig. 5C). These findings are consistent with the ChIP-chip data obtained from the rpb1-1 strain that provided evidence for PIC assembly in the absence to nucleosome depletion (Fig. 3B).

TFIIH is acquired downstream rather than at the promoter of many SAGA-directed transcription complexes

The promoter regions in cluster 20 (Fig. 5A, left panel) incorporated Pol II upon heat shock, but recruitment of Ssl2 (TFIIH) generally did not occur. Consistent with this, expression of genes in cluster 20 generally do not rely on the Kin28 subunit of TFIIH $\left(p<10^{-8}\right)$ (entry 376 in Supplementary Table S2). Kin28 transitions Pol II into a transcribing enzyme by phosphorylating the C-terminal domain of Pol II (Feaver et al. 1994; Akoulitchev et al. 1995; Cismowski et al. 1995). Genes in cluster 20 were also strongly biased toward SAGA regulation rather than TFIID $\left(p<10^{-16}\right)$ (entry 562 in Supplementary Table S2), suggesting that the lack of TFIIH recruitment predominates in the SAGA assembly pathway. In contrast, we observed Ssl2 (TFIIH) dissociation from the TFIID-dominated promoter regions in cluster 18. Taken together, these findings indicate a general trend whereby TFIIH is recruited to TFIID-assembled PICs but not stably recruited to heat-shock-induced SAGA-assembled PICs. In the latter case, TFIIH might not be essential for transcription initiation, as has been demonstrated at selected promoters in vitro (Makela et al. 1995; Chen and Zhou 1999|, at promoters regulated by Heat-Shock Factor 1 in vivo (Lee and Lis 1998; Sakurai et al. 2003), and at many genes throughout the genome (Holstege et al. 1998). Most other genes nevertheless require TFIIH (Holstege et al. 1998).

Inasmuch as TFIIH is directly linked to DNA repair including during the elongation phase of transcription (Drapkin et al. 1994), we investigated whether those genes that did not load TFIIH at the promoter might instead load TFIIH further downstream. This was accomplished by examining changes in Ssl2 (TFIIH) occupancy near the $3^{\prime}$ end of the genes in cluster 20 . Indeed, many of these genes displayed increased Ssl2 (TFIIH) occupancy near the $3^{\prime}$ end of the gene relative to the $5^{\prime}$ end (Fig. 5A, cf. columns 6 vs. 14 for cluster 20). This increased occupancy is unlikely to be attributable to binding to promoters of the next downstream gene since they displayed little or no increase in GTF occupancy or changes in gene expression (Fig. 5A, columns 16,17). One interpretation of the data is that many heat-shock-induced genes acquire TFIIH somewhere along the body of the gene rather than at the 5' end during PIC assembly, while others in this class do not acquire TFIIH at all.

\section{TFIIF remains at promoter regions}

The finding of TFIIH at the end of many cluster 20 genes rather than at the beginning prompted us to more thoroughly examine the distribution of Pol II and GTFs at the end of genes. We selected intergenic regions that were downstream from two convergent genes so that they were unlikely to contain promoters. These regions were also filtered to be in the top 10 percentile for increased Rpo21/Rpb1 occupancy upon heat shock. We then measured the median GTF, Pol II, and histone occupancy changes in these regions. As shown in Figure 5D, these downstream regions were enriched with Pol II (Rpo21, $\mathrm{Rpb2}$, Rpb7) and deficient in H2A.Z, as expected. As noted earlier, while H2A.Z is highly enriched in promoter regions, lower levels are also present downstream of genes (Fig. 1B). Downstream regions that contained Pol II were also highly enriched with TFIIH, reconfirming that TFIIH tracks with Pol II. No other GTF, including TFIIF, was enriched in the downstream regions. Others have reported similar findings on TFIIF at selected genes (Zawel et al. 1995; Krogan et al. 2002; Pokholok et al. 2002). Our findings demonstrate that TFIIF generally does not follow Pol II to the end of most genes. These results are in complete agreement with TFIIF's role in early initiation events including suppressing abortive initiation and the prevention of premature arrest of early elongation intermediates (Yan et al. 1999).

\section{Partial PICs assemble into full PICs in response to oxidative stress}

Why do cells tear down their transcription machinery at some promoters and rebuild it at others in response to heat shock? Certainly several heat-shock-induced genes protect the cell against the potential damaging effects of high temperatures and other stresses (Gasch et al. 2000). The shutdown of ribosomal protein genes could be rationalized as a means of curtailing protein synthesis and thus cell growth during harsh conditions. However, upon heat shock (sudden shift from $25^{\circ} \mathrm{C}$ to $37^{\circ} \mathrm{C}$ ), protein synthesis continues unabated, and ribosomal protein levels remain high (Gorenstein and Warner 1976). Moreover, metabolic activity and cell growth accelerate, rather than arrest. Consistent with this, a large fraction of heat-shock-induced genes appear to be geared toward meeting altered energy demands (Gasch et al. 2000). The transient disassembly of the transcription machinery at ribosomal protein genes during heat shock might provide a pool of transcription proteins needed for inducing other genes, as ribosomal protein genes represent a large group with a high occupancy of transcription proteins. Rapid transcriptional shutdown might also represent an imme- 
diate "knee-jerk" response to any environmental perturbation. If the cell subsequently finds the perturbation to be innocuous, the response is transient with little impact on the proteome. If not, the cell may be better prepared to arrest growth and cope with the stress.

What then is the regulatory logic behind redistributing sequence-specific activators (cluster 12), partially remodeling chromatin (cluster 17), and partially assembling PICs (clusters 8 and 21) in response to heat shock without substantially affecting gene expression? Might this be a preparatory response toward additional stresses? As diagrammed in Figure 6A, these clusters are highly related to each other, which indicates that many of the same genes redistribute sequence-specific and chromatin regulators, and partially assemble PICs upon heat shock without substantially altering gene expression. The genes in these clusters and the sequence-specific regulators that bind to them tend to be involved in energy metabolism, cell cycle control, cell growth, and stress responses. Conceivably, these genes might be poised at the point of partial PIC assembly in preparation for facing additional stresses.

To address this possibility, we examined whether the genes in cluster 8, which assemble into partial PICs, could be driven to assemble full PICs when exposed to a second stress. Cells were heat-shocked and at the same time treated with or without a second stress; then changes in occupancy of Pol II (Rpo21/Rpb1) and H2A.Z (Htz1) were measured. These stresses, which include hydrogen peroxide (oxidative stress), dithiothreitol (possibly cell wall damage), diamide (composite of stresses), nitrogen depletion, and ethanol, were chosen for their well-documented effects on the yeast transcriptome (Gasch et al. 2000; Causton et al. 2001; Harbison et al. 2004).

Interestingly, cluster 8 genes loaded Pol II and unloaded H2A.Z upon exposure to heat shock when combined with hydrogen peroxide but not diamide (Fig. 6B, left panel). The same trend was observed using standard ChIP assays (Fig. 6C). Peroxide treatment alone without heat shock did not cause these genes to be expressed (data not shown) and thus were likely to require both heat shock and peroxide for full assembly. Other stresses generated responses that were not much different from their controls (Fig. 6B, left panel). Thus, many of the partial PICs in cluster 8 appear to be poised to respond to oxidative stress but not to several other types of stress. Not all clusters responded to hydrogen peroxide like cluster 8. For example, cluster 4 genes that undergo full PIC assembly with heat shock alone were largely unaffected by the addition of hydrogen peroxide and other stresses (Fig. 6B, right panel).

A high level of respiration during continued growth at $37^{\circ} \mathrm{C}$ generates oxidative stress, which contributes substantially to cell death at elevated temperatures (Davidson and Schiestl 2001). If the partial PICs that are rapidly assembled at cluster 8 genes are poised to respond to oxidative stress, then continued incubation at $37^{\circ} \mathrm{C}$ should lead to a gradual conversion of these partial PICs into full PICs, as oxygen free-radical levels gradually in- crease. Consistent with this, a slow accumulation of Pol II (Rpo21/Rpb1) and a slow loss of H2A.Z (Htz1) were observed at cluster 8 genes over a course of $2 \mathrm{~h}$ (Fig. 6D, left panel). In contrast, cluster 4 genes underwent rapid and transient PIC assembly (Fig. 6D, right panel), which mirrored the transcriptional response reported elsewhere (Gasch et al. 2000; Causton et al. 2001). A similar but inverted pattern was observed for heat-repressed genes (data not shown). Taken together, these findings suggest that when yeast cells respond to one stress they also prepare themselves, through partial PIC assembly, for additional stresses that either accompany or result from the primary stress.

\section{Discussion}

As with any signaling event, cells respond to an environmental change by reprogramming their genome. Transcriptional activators find their target genes through interactions with specific DNA sequences. Chromatin might facilitate the search by hiding nontarget DNA sequences and using compositionally distinct nucleosomes such as H2A.Z to mark promoters. The transcription machinery, including the GTFs TFIIA, TFIIB, TFIID, TFIIE, and TFIIF, assembles in the midst of these nucleosomes, either in constitutively nucleosome-free regions or directly on nucleosomal DNA (Fig. 7). Therefore, nucleosome ejection is not a prerequisite for PIC assembly at most promoters, although other types of remodeling are not excluded. For example, SWI/SNF remodels chromatin at the SUC2 promoter without a requirement for transcription (Hirschhorn et al. 1992; Sharma et al. 2003). Moreover, we have identified a collection of promoter-containing intergenic regions that undergo some loss of histone $\mathrm{H} 1$ and standard nucleosomes (i.e., not H2A.Z), but without becoming activated (cluster 17 in Fig. 4B).

The presence of certain nucleosomes at promoter regions may, in fact, be stimulatory in that H4-acetylated nucleosomes might enhance PIC assembly via interactions with bromodomains associated with TFIID and SAGA. When a transcriptionally active Pol II arrives, H2A.Z nucleosomes are ejected from the promoter. A similar prerequisite for GTFs and an active Pol II to remodel chromatin was first observed at the RNR3 promoter (Sharma et al. 2003).

Our discovery of partial PICs in vivo indicates that GTF assembly at many promoters precedes the arrival of Pol II, and that Pol II (and/or TFIIH) recruitment can be a separately regulated event. The ability to convert partial PICs into full PICs, and reciprocally full PICs into partial PICs, provides strong evidence that partial PICs are novel regulated intermediates that are widespread throughout the genome. Partial PIC assembly might provide a mechanism for converging distinct signaling pathways (e.g., heat and oxidative stress) at target genes, where one signal triggers partial PIC assembly and the other triggers Pol II recruitment. The ability to simultaneously respond to one signal/stress and prepare for another that typically co-occurs in nature might contribute 

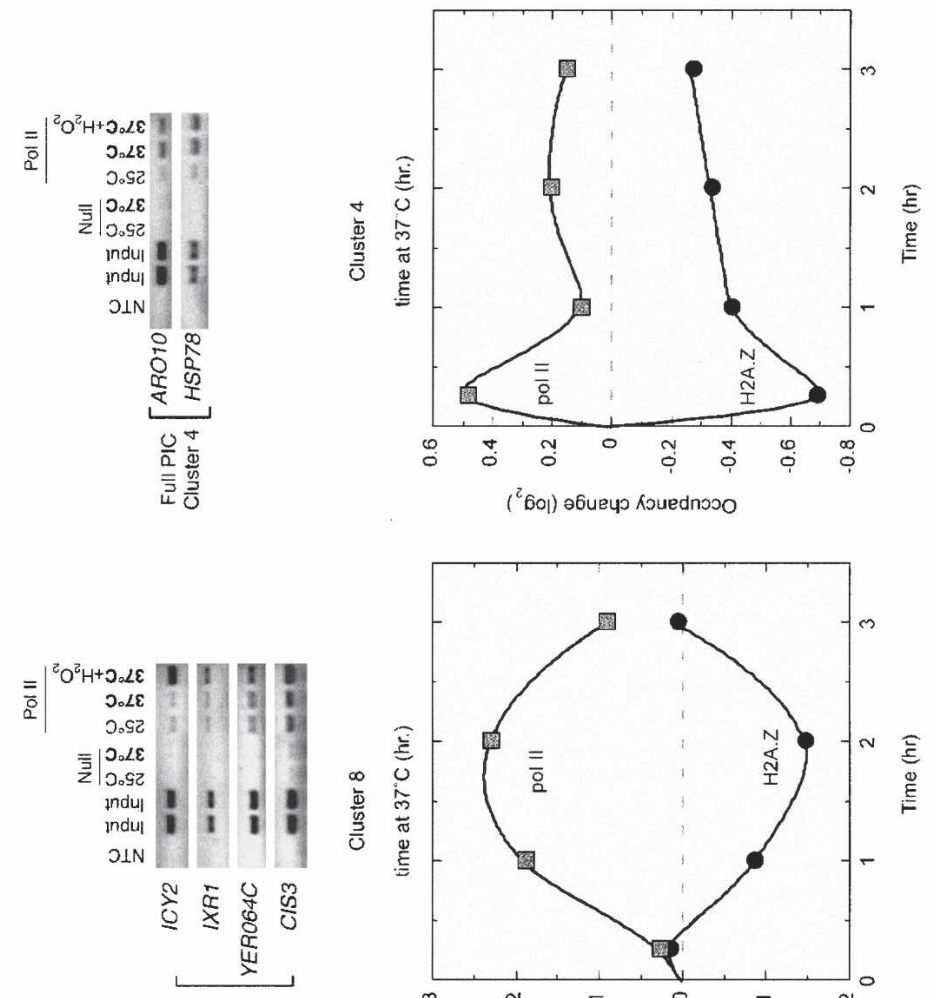

$0 \quad \frac{0}{20}$

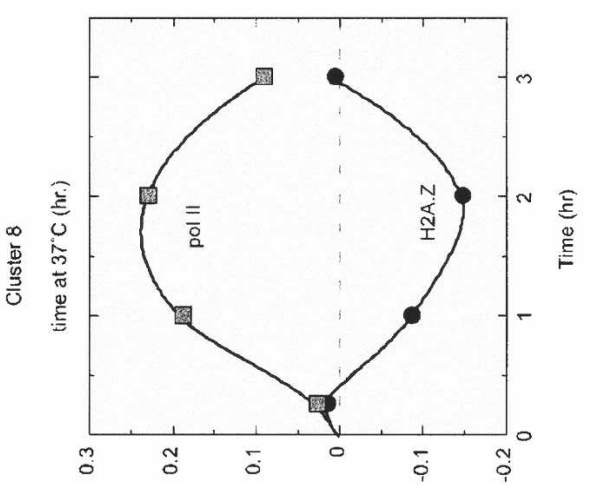

( ${ }^{2}$ 6이) a6ิueys রouednoxo

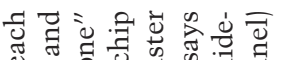

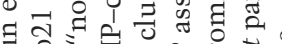

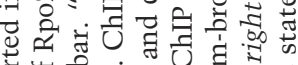

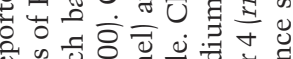

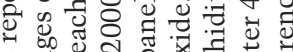

월

of

范

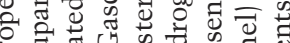

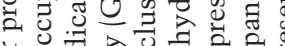
$\Rightarrow 0$ त्रे

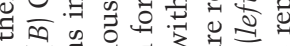

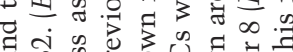
के के की

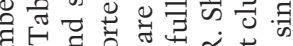
入 चै 氙

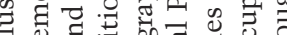
Uี क्ञ

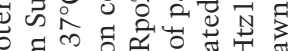

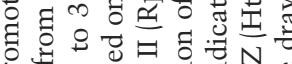
号焉

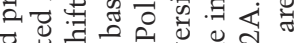

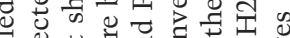

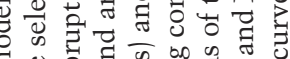

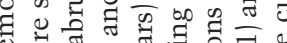

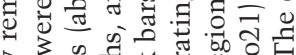
त) के स्ञ

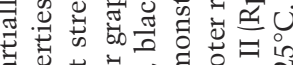

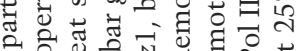

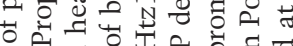

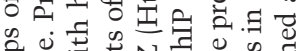

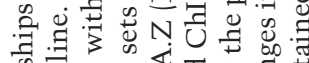
की .

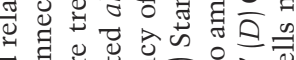
उ 010

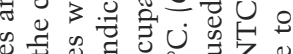

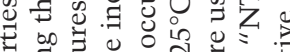

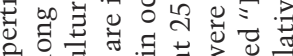

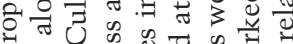
ज्ञ 0 के

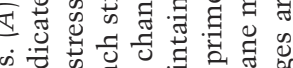
نे के

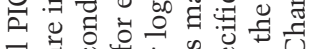

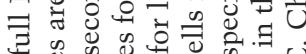

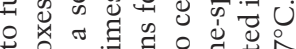
की

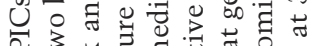

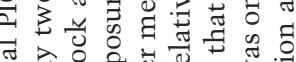

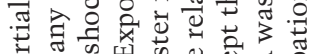

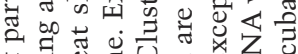

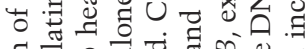

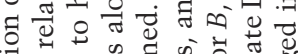
कै

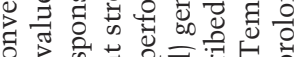
ठี क्षे क्षे

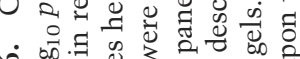

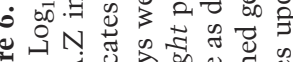

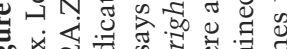

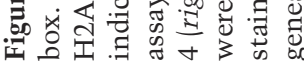




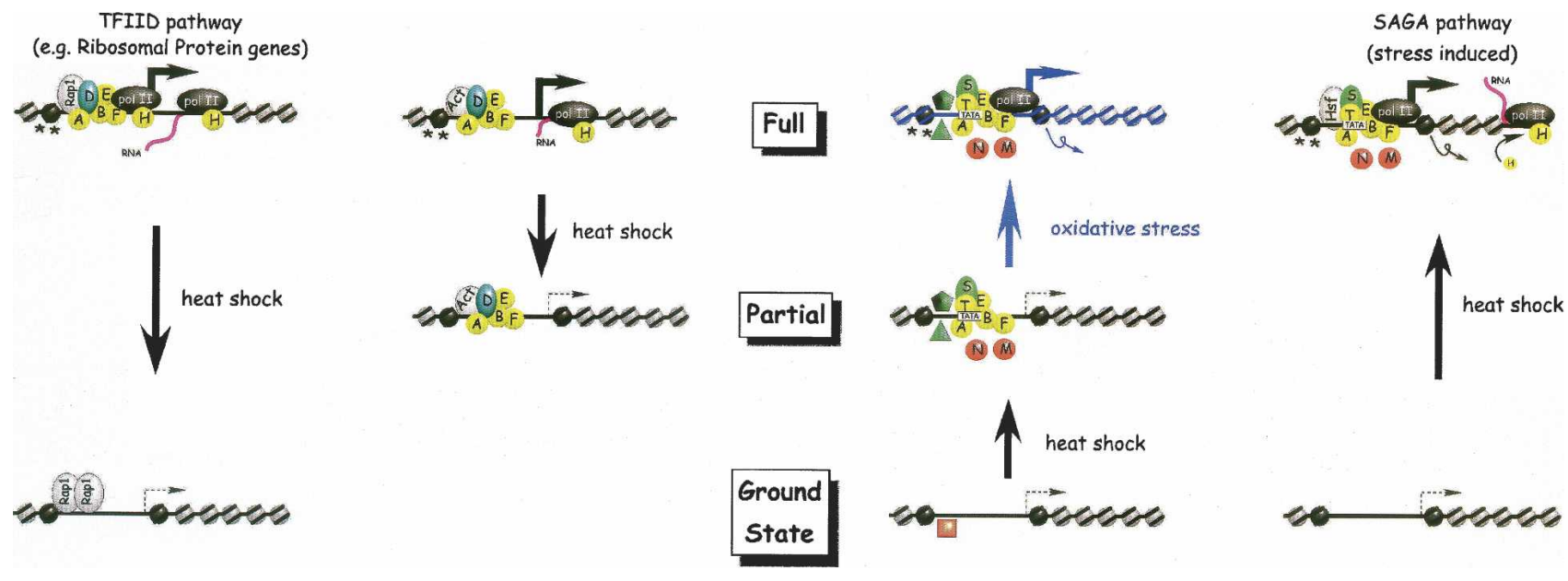

Figure 7. A model for full and partial PICs that are widespread throughout the yeast genome. Models roughly correspond to the behaviors of clusters 18-21 (Fig. 5A), as drawn from left to right. The bottom illustrations represent the inactive ground state chromatin structure. Upon heat shock, partial PIC assembly occurs at some genes (third pathway) and full assembly at others (fourth pathway). At many highly active housekeeping genes such as the ribosomal protein genes, heat shock leads to a transient return to the ground state primarily through the TFIID pathway (first pathway). At other genes, the PIC partly disassembles (second pathway). (A) TFIIA; (B) TFIIB; (D) TFIID; (E) TFIIE; (F) TFIIF; (H) TFIIH; (M) Mot1; (N) NC2; (S) SAGA; $\left(^{\star \star}\right.$ ) H3 acetylation. The depicted partial PICs represent a minimal composition. Other regulatory proteins including a variety of sequence-specific regulators are likely to be present, and some of the proteins shown could be absent at certain promoters.

to the phenomenon of cross-protection, where sublethal doses of one stress provide enhanced protection for another stress (Collinson and Dawes 1992).

\section{Materials and methods}

Strains and data

C-terminally TAP tagged Saccharomyces cerevisiae S288C strains were purchased from Open Biosystems and have the

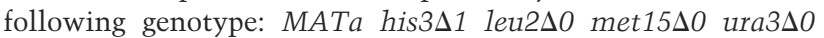
(Ghaemmaghami et al. 2003). TAP tags were transferred from these strains into the rpb1-1 strain (Nonet et al. 1987) by homologous recombination. Strain BY4741 was used for the H3K9,14Ac, polyclonal TBP, Pol II 8WG16, and null ChIP. Gene expression data and null, Spt3, Spt15, Taf1, Mot1, and Bdf1 ChIP-chip data were from Zanton and Pugh (2004). Hsfl data were from Hahn et al. (2004). References to other data set comparisons are provided in Supplementary Table S2. Raw data are accessible at GEO (http://www.ncbi.nlm.nih.gov/geo) under series accession numbers GSE1373 and GSE2488.

\section{ChIP-chip}

Assays were performed as described previously (Zanton and Pugh 2004). Briefly, 100-mL cell cultures were grown to $\mathrm{OD}_{600}=0.8$ in YPD at $25^{\circ} \mathrm{C}$. All TAP-tagged strains grew at approximately wild-type rates. $r p b 1-1$ strains grew more slowly. Cultures were rapidly shifted to $37^{\circ} \mathrm{C}$ or mock-shifted to $25^{\circ} \mathrm{C}$ for 15 min. rpb1-1 strains were shifted for $45 \mathrm{~min}$. Cultures were diluted with a chilled formaldehyde solution, resulting in a $1 \%$ final concentration at $25^{\circ} \mathrm{C}$. Incubation was continued for $2 \mathrm{~h}$ at $25^{\circ} \mathrm{C}$. Harvested cells were disrupted with zirconium beads, and the chromatin pellet was washed prior to sonication. Sheared chromatin was immunoprecipitated with IgG-Sepharose, or by an immobilized protein-specific antibody. This enriched ChIP DNA was amplified by either 20 or 25 rounds of
$\mathrm{PCR}$, as indicated in Supplementary Table S1. The $37^{\circ} \mathrm{C}$ samples were then Cy5-labeled, and the $25^{\circ} \mathrm{C}$ samples were Cy3-labeled. These dyes were swapped for independent replicates, which ranged from two to eight for each temperature. The $37^{\circ} \mathrm{C}$ and $25^{\circ} \mathrm{C}$ samples were cohybridized to microarrays containing $>6000$ spotted PCR probes spanning nearly every intergenic region. Conventional PCR was routinely used to verify the hybridization results.

For cultures that received additional stresses (Fig. 6), both test and reference samples were treated exactly as described above (15-min heat shock). In addition, the test samples were either exposed or mock-exposed to one additional stress at $37^{\circ} \mathrm{C}$, which included $4 \mathrm{mM}$ hydrogen peroxide or $1.5 \mathrm{mM}$ diamide over the original $15 \mathrm{~min}, 2.5 \mathrm{mM}$ dithiothreitol for $3 \mathrm{~h}, 2 \%$ ethanol in place of glucose for $8 \mathrm{~h}$, or nitrogen-depleted media (minimal media lacking amino acids and ammonium sulfate and supplemented with $20 \mathrm{mg} / \mathrm{L}$ uracil, $0.02 \mathrm{mg} / \mathrm{L}$ methionine, $0.1 \mathrm{mg} / \mathrm{L}$ leucine, and 2\% glucose) in place of YPD for $8 \mathrm{~h}$ (Gasch et al. 2000). Culture densities were maintained between $\mathrm{OD}_{600} 0.8$ and 1.0 by periodic addition of the same sterile media.

\section{Data normalization}

Details are presented in the Supplemental Material. Briefly, occupancy levels at $25^{\circ} \mathrm{C}$ and $37^{\circ} \mathrm{C}$ were normalized to the corresponding values for negative control samples ("Hxk1" when 20 PCR cycles were used during nonspecific sample amplification, and "Null" when 25 PCR cycles were used), which represent amplified background DNA that contaminates the ChIP DNA. The use of Hxk1 (a cytoplasmic hexokinase that is not expected to bind DNA) versus null (no TAP tag) was arbitrary, with both generating similar low nonspecific ChIP signals that are reflective of the expected lack of DNA binding. Data were then converted to a $\log _{2}$ scale and centered to the median value of all nonpromoter regions within the same data set (identified as "T- $\mathrm{T}$ " in Supplementary Table S1). Supplementary Table S1 also reports the median value for multiple repeats. In Figure 1B only, the data were centered to the nonpromoter regions in the 
control set rather than within the same data set. Changes in occupancy represent $\log _{2}$ ratios of $37^{\circ} \mathrm{C}$ to $25^{\circ} \mathrm{C}$ data derived from cohybridized samples, and were centered as described above. An abbreviated formula of the occupancy and changes in occupancy follows:

Occupancy $=\log _{2}\left(25^{\circ} \mathrm{C}\right.$ value/"null" or "Hxk1" control value $)$;

Occupancy change $=\log _{2}\left(37^{\circ} \mathrm{C} / 25^{\circ} \mathrm{C}\right.$ ratio in promoter regions) $-\log _{2}$ (median of all $37^{\circ} \mathrm{C} / 25^{\circ} \mathrm{C}$ ratios in nonpromoter regions).

\section{Acknowledgments}

We thank T. Mavrich, M. Durant, D. Gilmour, K. Huisinga, J. Reese, S. Tan, and Y. Wang for many helpful discussions, and T. Mavrich for much technical assistance. This work was supported by NIH grant GM59055 and NSF grant 0425662 .

\section{References}

Akoulitchev, S., Makela, T.P., Weinberg, R.A., and Reinberg, D. 1995. Requirement for TFIIH kinase activity in transcription by RNA polymerase II. Nature 377: 557-560.

Basehoar, A.D., Zanton, S.J., and Pugh, B.F. 2004. Identification and distinct regulation of yeast TATA box-containing genes. Cell 116: 699-709.

Bell, S.D., Brinkman, A.B., van der Oost, J., and Jackson, S.P. 2001. The archaeal TFIIE $\alpha$ homologue facilitates transcription initiation by enhancing TATA-box recognition. EMBO Rep. 2: 133-138.

Bernstein, B.E., Liu, C.L., Humphrey, E.L., Perlstein, E.O., and Schreiber, S.L. 2004. Global nucleosome occupancy in yeast. Genome Biol. 5: R62.

Bhaumik, S.R. and Green, M.R. 2001. SAGA is an essential in vivo target of the yeast acidic activator Gal4p. Genes \& Dev. 15: 1935-1945.

Boeger, H., Griesenbeck, J., Strattan, J.S., and Kornberg, R.D. 2004. Removal of promoter nucleosomes by disassembly rather than sliding in vivo. Mol. Cell 14: 667-673.

Bryant, G.O. and Ptashne, M. 2003. Independent recruitment in vivo by Gal4 of two complexes required for transcription. Mol. Cell 11: 1301-1309.

Buratowski, S., Hahn, S., Guarente, L., and Sharp, P.A. 1989. Five intermediate complexes in transcription initiation by RNA polymerase II. Cell 56: 549-561.

Causton, H.C., Ren, B., Koh, S.S., Harbison, C.T., Kanin, E., Jennings, E.G., Lee, T.I., True, H.L., Lander, E.S., and Young, R.A. 2001. Remodeling of yeast genome expression in response to environmental changes. Mol. Biol. Cell 12: 323337.

Chen, D. and Zhou, Q. 1999. Tat activates human immunodeficiency virus type 1 transcriptional elongation independent of TFIIH kinase. Mol. Cell. Biol. 19: 2863-2871.

Cheng, J.X., Floer, M., Ononaji, P., Bryant, G., and Ptashne, M. 2002. Responses of four yeast genes to changes in the transcriptional machinery are determined by their promoters. Curr. Biol. 12: 1828-1832.

Cismowski, M.J., Laff, G.M., Solomon, M.J., and Reed, S.I. 1995. KIN28 encodes a C-terminal domain kinase that controls mRNA transcription in Saccharomyces cerevisiae but lacks cyclin-dependent kinase-activating kinase (CAK) activity. Mol. Cell. Biol. 15: 2983-2992.

Collinson, L.P. and Dawes, I.W. 1992. Inducibility of the response of yeast cells to peroxide stress. J. Gen. Microbiol. 138: 329-335.
Conaway, J.W., Reines, D., and Conaway, R.C. 1990. Transcription initiated by RNA polymerase II and purified transcription factors from liver. Cooperative action of transcription factors $\tau$ and $\varepsilon$ in initial complex formation. J. Biol. Chem. 265: 7552-7558.

Conaway, J.W., Bradsher, J.N., and Conaway, R.C. 1992. Mechanism of assembly of the RNA polymerase II preinitiation complex. Transcription factors $\delta$ and $\varepsilon$ promote stable binding of the transcription apparatus to the initiator element. $J$. Biol. Chem. 267: 10142-10148.

Cosma, M.P., Panizza, S., and Nasmyth, K. 2001. Cdk1 triggers association of RNA polymerase to cell cycle promoters only after recruitment of the mediator by SBF. Mol. Cell 7: 12131220.

Davidson, J.F. and Schiestl, R.H. 2001. Mitochondrial respiratory electron carriers are involved in oxidative stress during heat stress in Saccharomyces cerevisiae. Mol. Cell. Biol. 21: 8483-8489.

Drapkin, R., Reardon, J.T., Ansari, A., Huang, J.C., Zawel, L., Ahn, K., Sancar, A., and Reinberg, D. 1994. Dual role of TFIIH in DNA excision repair and in transcription by RNA polymerase II. Nature 368: 769-772.

Dubrovskaya, V., Lavigne, A.C., Davidson, I., Acker, J., Staub, A., and Tora, L. 1996. Distinct domains of hTAFIII00 are required for functional interaction with transcription factor TFIIF $\beta$ (RAP30) and incorporation into the TFIID complex. EMBO T. 15: 3702-3712.

Eisen, M.B., Spellman, P.T., Brown, P.O., and Botstein, D. 1998. Cluster analysis and display of genome-wide expression patterns. Proc. Nat1. Acad. Sci. 95: 14863-14868.

Ercan, S., Carrozza, M.J., and Workman, J.L. 2004. Global nucleosome distribution and the regulation of transcription in yeast. Genome Biol. 5: 243.

Fan, X., Chou, D.M., and Struhl, K. 2006. Activator-specific recruitment of Mediator in vivo. Nat. Struct. Mol. Biol. 13: 117-120.

Fang, S.M. and Burton, Z.F. 1996. RNA polymerase II-associated protein (RAP) 74 binds transcription factor (TF) IIB and blocks TFIIB-RAP30 binding. I. Biol. Chem. 271: 1170311709.

Feaver, W.J., Svejstrup, J.Q., Henry, N.L., and Kornberg, R.D. 1994. Relationship of CDK-activating kinase and RNA polymerase II CTD kinase TFIH/TFIIK. Cell 79: 1103-1109.

Flores, O., Lu, H., Killeen, M., Greenblatt, J., Burton, Z.F., and Reinberg, D. 1991. The small subunit of transcription factor IIF recruits RNA polymerase II into the preinitiation complex. Proc. Natl. Acad. Sci. 88: 9999-10003.

Flores, O., Lu, H., and Reinberg, D. 1992. Factors involved in specific transcription by mammalian RNA polymerase II. Identification and characterization of factor IIH. I. Biol. Chem. 267: 2786-2793.

Freidkin, I. and Katcoff, D.J. 2001. Specific distribution of the Saccharomyces cerevisiae linker histone homolog HHOlp in the chromatin. Nucleic Acids Res. 29: 4043-4051.

Gasch, A.P., Spellman, P.T., Kao, C.M., Carmel-Harel, O., Eisen, M.B., Storz, G., Botstein, D., and Brown, P.O. 2000. Genomic expression programs in the response of yeast cells to environmental changes. Mol. Biol. Cell 11: 4241-4257.

Ghaemmaghami, S., Huh, W.K., Bower, K., Howson, R.W., Belle, A., Dephoure, N., O'Shea, E.K., and Weissman, J.S. 2003. Global analysis of protein expression in yeast. Nature 425: 737-741.

Gorenstein, C. and Warner, J.R. 1976. Coordinate regulation of the synthesis of eukaryotic ribosomal proteins. Proc. Nat1. Acad. Sci. 73: 1547-1551.

Guillemette, B., Bataille, A.R., Gevry, N., Adam, M., Blan- 
chette, M., Robert, F., and Gaudreau, L. 2005. Variant histone H2A.Z is globally localized to the promoters of inactive yeast genes and regulates nucleosome positioning. PLOS Biol. 3: e384.

Ha, I., Roberts, S., Maldonado, E., Sun, X., Kim, L.U., Green, M., and Reinberg, D. 1993. Multiple functional domains of human transcription factor IIB: Distinct interactions with two general transcription factors and RNA polymerase II. Genes \& Dev. 7: 1021-1032.

Hahn, J.S., Hu, Z., Thiele, D.J., and Iyer, V.R. 2004. Genomewide analysis of the biology of stress responses through heat shock transcription factor. Mol. Cell. Biol. 24: 5249-5256.

Harbison, C.T., Gordon, D.B., Lee, T.I., Rinaldi, N.J., Macisaac, K.D., Danford, T.W., Hannett, N.M., Tagne, J.B., Reynolds, D.B., Yoo, J., et al. 2004. Transcriptional regulatory code of a eukaryotic genome. Nature 431: 99-104.

Hassan, A.H., Neely, K.E., Vignali, M., Reese, J.C., and Workman, J.L. 2001. Promoter targeting of chromatin-modifying complexes. Front. Biosci. 6: D1054-D1064.

Hirschhorn, J.N., Brown, S.A., Clark, C.D., and Winston, F. 1992. Evidence that SNF2/SWI2 and SNF5 activate transcription in yeast by altering chromatin structure. Genes \& Dev. 6: 2288-2298.

Holstege, F.C., Jennings, E.G., Wyrick, J.J., Lee, T.I., Hengartner, C.J., Green, M.R., Golub, T.R., Lander, E.S., and Young, R.A. 1998. Dissecting the regulatory circuitry of a eukaryotic genome. Cell 95: 717-728.

Huisinga, K.L. and Pugh, B.F. 2004. A genome-wide housekeeping role for TFIID and a highly regulated stress-related role for SAGA in Saccharomyces cerevisiae. Mol. Cell 13: 573585.

Joliot, V., Demma, M., and Prywes, R. 1995. Interaction with RAP74 subunit of TFIIF is required for transcriptional activation by serum response factor. Nature 373: 632-635.

Killeen, M., Coulombe, B., and Greenblatt, J. 1992. Recombinant TBP, transcription factor IIB, and RAP30 are sufficient for promoter recognition by mammalian RNA polymerase II. J. Biol. Chem. 267: 9463-9466.

Kornberg, R.D. 2005. Mediator and the mechanism of transcriptional activation. Trends Biochem. Sci. 30: 235-239.

Krogan, N.J., Kim, M., Ahn, S.H., Zhong, G., Kobor, M.S., Cagney, G., Emili, A., Shilatifard, A., Buratowski, S., and Greenblatt, J.F. 2002. RNA polymerase II elongation factors of Saccharomyces cerevisiae: A targeted proteomics approach. Mol. Cell. Biol. 22: 6979-6992.

Kuras, L., Kosa, P., Mencia, M., and Struhl, K. 2000. TAF-containing and TAF-independent forms of transcriptionally active TBP in vivo. Science 288: 1244-1248.

Langelier, M.F., Forget, D., Rojas, A., Porlier, Y., Burton, Z.F., and Coulombe, B. 2001. Structural and functional interactions of transcription factor (TF) IIA with TFIIE and TFIIF in transcription initiation by RNA polymerase II. J. Biol. Chem. 276: 38652-38657.

Lee, D. and Lis, J.T. 1998. Transcriptional activation independent of TFIIH kinase and the RNA polymerase II mediator in vivo. Nature 393: 389-392.

Lee, T.I., Causton, H.C., Holstege, F.C., Shen, W.C., Hannett, N., Jennings, E.G., Winston, F., Green, M.R., and Young, R.A. 2000. Redundant roles for the TFIID and SAGA complexes in global transcription. Nature 405: 701-704.

Lee, T.I., Rinaldi, N.J., Robert, F., Odom, D.T., Bar-Joseph, Z., Gerber, G.K., Hannett, N.M., Harbison, C.T., Thompson, C.M., Simon, I., et al. 2002. Transcriptional regulatory networks in Saccharomyces cerevisiae. Science 298: 799-804.

Lee, C.K., Shibata, Y., Rao, B., Strahl, B.D., and Lieb, J.D. 2004. Evidence for nucleosome depletion at active regulatory re- gions genome-wide. Nat. Genet. 36: 900-905.

Levine, M. and Tjian, R. 2003. Transcription regulation and animal diversity. Nature 424: 147-151.

Li, B., Nierras, C.R., and Warner, J.R. 1999. Transcriptional elements involved in the repression of ribosomal protein synthesis. Mol. Cell. Biol. 19: 5393-5404.

Li, X.Y., Bhaumik, S.R., and Green, M.R. 2000. Distinct classes of yeast promoters revealed by differential TAF recruitment. Science 288: 1242-1244.

Li, B., Pattenden, S.G., Lee, D., Gutierrez, J., Chen, J., Seidel, C., Gerton, J., and Workman, J.L. 2005. Preferential occupancy of histone variant $\mathrm{H} 2 \mathrm{AZ}$ at inactive promoters influences local histone modifications and chromatin remodeling. Proc. Natl. Acad. Sci. 102: 18385-18390.

Lieb, J.D. and Clarke, N.D. 2005. Control of transcription through intragenic patterns of nucleosome composition. Cell 123: 1187-1190.

Lieb, J.D., Liu, X., Botstein, D., and Brown, P.O. 2001. Promoterspecific binding of Rap1 revealed by genome-wide maps of protein-DNA association. Nat. Genet. 28: 327-334.

Makela, T.P., Parvin, J.D., Kim, J., Huber, L.J., Sharp, P.A., and Weinberg, R.A. 1995. A kinase-deficient transcription factor TFIIH is functional in basal and activated transcription. Proc. Natl. Acad. Sci. 92: 5174-5178.

Martin, M.L., Lieberman, P.M., and Curran, T. 1996. Fos-Jun dimerization promotes interaction of the basic region with TFIIE-34 and TFIIF. Mol. Cell. Biol. 16: 2110-2118.

McEwan, I.J. and Gustafsson, J. 1997. Interaction of the human androgen receptor transactivation function with the general transcription factor TFIIF. Proc. Natl. Acad. Sci. 94: 84858490.

Narlikar, G.J., Fan, H.Y., and Kingston, R.E. 2002. Cooperation between complexes that regulate chromatin structure and transcription. Cell 108: 475-487.

Nonet, M., Scafe, C., Sexton, J., and Young, R. 1987. Eucaryotic RNA polymerase conditional mutant that rapidly ceases mRNA synthesis. Mol. Cell. Biol. 7: 1602-1611.

Orphanides, G., Lagrange, T., and Reinberg, D. 1996. The general transcription factors of RNA polymerase II. Genes \& Dev. 10: 2657-2683.

Patterton, H.G., Landel, C.C., Landsman, D., Peterson, C.L., and Simpson, R.T. 1998. The biochemical and phenotypic characterization of Hholp, the putative linker histone $\mathrm{H} 1$ of Saccharomyces cerevisiae. J. Biol. Chem. 273: 7268-7276.

Pokholok, D.K., Hannett, N.M., and Young, R.A. 2002. Exchange of RNA polymerase II initiation and elongation factors during gene expression in vivo. Mol. Cell 9: 799-809.

Raisner, R.M., Hartley, P.D., Meneghini, M.D., Bao, M.Z., Liu, C.L., Schreiber, S.L., Rando, O.J., and Madhani, H.D. 2005. Histone variant H2A.Z marks the $5^{\prime}$ ends of both active and inactive genes in euchromatin. Cell 123: 233-248.

Reinberg, D., Orphanides, G., Ebright, R., Akoulitchev, S., Carcamo, J., Cho, H., Cortes, P., Drapkin, R., Flores, O., Ha, I., et al. 1998. The RNA polymerase II general transcription factors: Past, present, and future. Cold Spring Harb. Symp. Quant. Biol. 63: 83-103.

Ruppert, S. and Tjian, R. 1995. Human TAFII250 interacts with RAP74: Implications for RNA polymerase II initiation. Genes \& Dev. 9: 2747-2755.

Sakurai, H., Hashikawa, N., Imazu, H., and Fukasawa, T. 2003. Carboxy-terminal region of the yeast heat shock factor contains two domains that make transcription independent of the TFIIH protein kinase. Genes Cells 8: 951-961.

Sauer, F., Fondell, J.D., Ohkuma, Y., Roeder, R.G., and Jackle, H. 1995. Control of transcription by Kruppel through interactions with TFIIB and TFIIE $\beta$. Nature 375: 162-164. 
Schwabish, M.A. and Struhl, K. 2004. Evidence for eviction and rapid deposition of histones upon transcriptional elongation by RNA polymerase II. Mol. Cell. Biol. 24: 10111-10117.

Sharma, V.M., Li, B., and Reese, J.C. 2003. SWI/SNF-dependent chromatin remodeling of RNR3 requires TAF(II)s and the general transcription machinery. Genes \& Dev. 17: 502-515.

Soutoglou, E. and Talianidis, I. 2002. Coordination of PIC assembly and chromatin remodeling during differentiation-induced gene activation. Science 295: 1901-1904.

Spellman, P.T., Sherlock, G., Zhang, M.Q., Iyer, V.R., Anders, K., Eisen, M.B., Brown, P.O., Botstein, D., and Futcher, B. 1998. Comprehensive identification of cell cycle-regulated genes of the yeast Saccharomyces cerevisiae by microarray hybridization. Mol. Biol. Cell 9: 3273-3297.

Struhl, K. 1999. Fundamentally different logic of gene regulation in eukaryotes and prokaryotes. Cell 98: 1-4.

Tong, X., Drapkin, R., Yalamanchili, R., Mosialos, G., and Kieff, E. 1995. The Epstein-Barr virus nuclear protein 2 acidic domain forms a complex with a novel cellular coactivator that can interact with TFIIE. Mol. Cell. Biol. 15: 4735-4744.

Yan, Q., Moreland, R.J., Conaway, J.W., and Conaway, R.C. 1999. Dual roles for transcription factor IIF in promoter escape by RNA polymerase II. I. Biol. Chem. 274: 3566835675.

Yokomori, K., Verrijzer, C.P., and Tjian, R. 1998. An interplay between TATA box-binding protein and transcription factors IIE and IIA modulates DNA binding and transcription. Proc. Natl. Acad. Sci. 95: 6722-6727.

Yuan, G.C., Liu, Y.J., Dion, M.F., Slack, M.D., Wu, L.F., Altschuler, S.J., and Rando, O.J. 2005. Genome-scale identification of nucleosome positions in S. cerevisiae. Science 309: 626630.

Zanton, S.J. and Pugh, B.F. 2004. Changes in genomewide occupancy of core transcriptional regulators during heat stress. Proc. Nat1. Acad. Sci. 101: 16843-16848.

Zawel, L., Kumar, K.P., and Reinberg, D. 1995. Recycling of the general transcription factors during RNA polymerase II transcription. Genes \& Dev. 9: 1479-1490.

Zhang, H., Roberts, D.N., and Cairns, B.R. 2005. Genome-wide dynamics of Htz1, a histone H2A variant that poises repressed/basal promoters for activation through histone loss. Cell 123: 219-231.

Zhu, A. and Kuziora, M.A. 1996. Homeodomain interaction with the $\beta$ subunit of the general transcription factor TFIIE. J. Biol. Chem. 271: 20993-20996. 


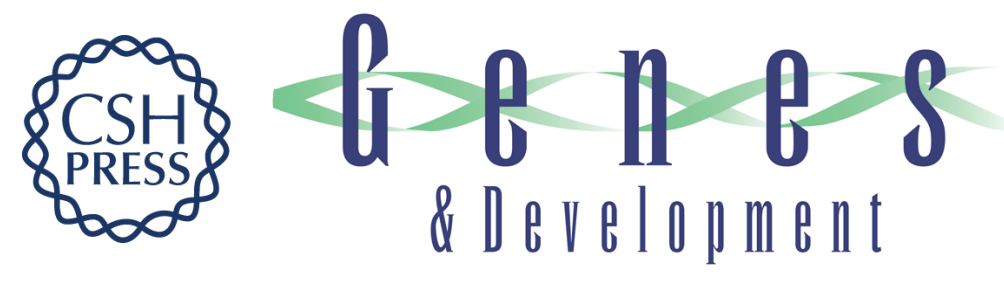

\section{Full and partial genome-wide assembly and disassembly of the yeast transcription machinery in response to heat shock}

Sara J. Zanton and B. Franklin Pugh

Genes Dev. 2006, 20:

Access the most recent version at doi:10.1101/gad.1437506

Supplemental http://genesdev.cshlp.org/content/suppl/2006/08/02/20.16.2250.DC1
Material

References This article cites 77 articles, 42 of which can be accessed free at:

http://genesdev.cshlp.org/content/20/16/2250.full.html\#ref-list-1

License

Email Alerting Receive free email alerts when new articles cite this article - sign up in the box at the top

Service

right corner of the article or click here.

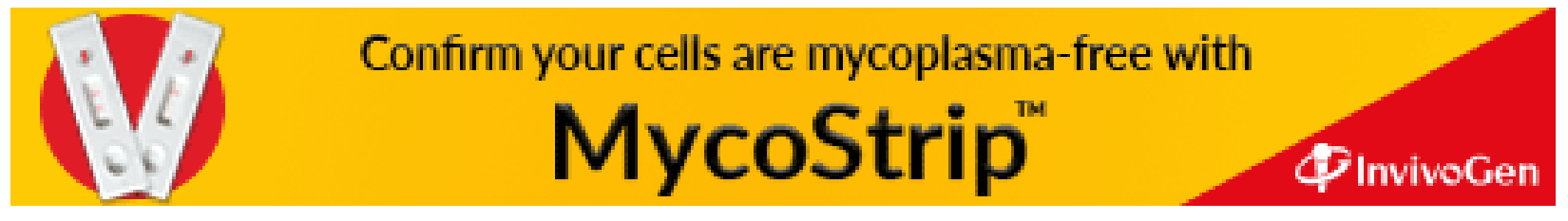

